TRANSACTIONS OF THE

AMERICAN MATHEMATICAL SOCIETY

Volume 353, Number 5, Pages 1885-1906

S 0002-9947(01)02695-2

Article electronically published on January 16, 2001

\title{
GEOMETRIC INTERPRETATION OF TIGHT CLOSURE AND TEST IDEALS
}

\author{
NOBUO HARA
}

\begin{abstract}
We study tight closure and test ideals in rings of characteristic $p \gg 0$ using resolution of singularities. The notions of $F$-rational and $F$ regular rings are defined via tight closure, and they are known to correspond with rational and $\log$ terminal singularities, respectively. In this paper, we reformulate this correspondence by means of the notion of the test ideal, and generalize it to wider classes of singularities. The test ideal is the annihilator of the tight closure relations and plays a crucial role in the tight closure theory. It is proved that, in a normal $\mathbb{Q}$-Gorenstein ring of characteristic $p \gg 0$, the test ideal is equal to so-called the multiplier ideal, which is an important ideal in algebraic geometry. This is proved in more general form, and to do this we study the behavior of the test ideal and the tight closure of the zero submodule in certain local cohomology modules under cyclic covering. We reinterpret the results also for graded rings.
\end{abstract}

The notion of the tight closure of an ideal in a commutative ring of prime characteristic was defined by Hochster and Huneke [HH1] in terms of the asymptotic behavior of the ideal under iteration of the Frobenius map. Tight closure enables us to define the notions of $F$-regular rings [HH1] and $F$-rational rings [FW]. Namely, a ring of characteristic $p>0$ is called $F$-regular (resp. $F$-rational) if all ideals (resp. all ideals generated by a system of parameters) are tightly closed in all of its local rings.

Although these concepts are defined quite ring-theoretically, they have been suspected to have a mysterious correspondence with some classes of singularities in characteristic zero defined via resolution of singularities. Surprisingly, recent results by Smith [S2], Watanabe [W3], Mehta and Srinivas [MS], and the author Ha conclude that a ring in characteristic zero has at most rational (resp. log terminal) singularities if and only if its reduction modulo $p$ is $F$-rational (resp. $F$-regular and $\mathbb{Q}$-Gorenstein) for $p \gg 0$.

The aim of this paper is to generalize these results to wider classes of singularities. To do this we use a fairly standard technique of "reduction modulo $p$," starting from a singularity in characteristic zero. Let $(R, \mathfrak{m})$ be a $d$-dimensional normal local ring which is reduced from characteristic zero to characteristic $p \gg 0$, together with a resolution of singularities $f: X \rightarrow \operatorname{Spec} R$. When the non- $(F-)$ rational locus of $(R, \mathfrak{m})$ is isolated, we actually proved in $\mathrm{Ha}$ that the tight closure of the zero

Received by the editors July 27, 1999.

2000 Mathematics Subject Classification. Primary 13A35, 14B05; Secondary 13A02, 14B15.

Key words and phrases. Tight closure, test ideal, modulo $p$ reduction, multiplier ideal.

The author is partially supported by Grant for Special Research Project 98A-140, Waseda University, and Grant-in-Aid for Scientific Research No. 11740028, Japan. 
submodule in the top local cohomology module $H_{\mathfrak{m}}^{d}(R)$, denoted by $0_{H_{\mathfrak{m}}^{d}(R)}^{*}$, is described as

$$
0_{H_{\mathfrak{m}}^{d}(R)}^{*}=H^{d-1}\left(X, \mathcal{O}_{X}\right) .
$$

The length of the right-hand side of this formula is an important invariant of the singularity, called the geometric genus. An isolated normal singularity in characteristic zero is rational if and only if it is Cohen-Macaulay and its geometric genus is zero. Therefore the above formula generalizes the correspondence of $F$ rationality and rational singularity, because $(R, \mathfrak{m})$ is $F$-rational if and only if $R$ is Cohen-Macaulay and $0_{H_{\mathrm{m}}^{d}(R)}^{*}=0$.

This result leads us to further questions. First, we ask what happens when the non- $(F-)$ rational locus of $(R, \mathfrak{m})$ is not isolated. Second, how can we generalize the correspondence of $F$-regularity and log terminal singularity?

An answer to the first question is already found in the paper of Mehta and Srinivas [MS. They gave a cohomological description of $0_{H_{\mathrm{m}}^{d}(R)}^{*}$ without assuming the isolatedness of the non- $(F-)$ rational locus. We give a slightly different proof to this result, using the "Q্-divisor technique" developed in [Ha]. Then we reformulate it in a dual form in terms of the "parameter test submodule" of the canonical module, which is an analog of the notion of the test ideal [HH1].

The test ideal is the annihilator of tight closure relations, and plays an important role in the tight closure theory. A recent result of MacCrimmon $[\mathrm{Mc}$ implies that the test ideal $\tau(R)$ of a normal $\mathbb{Q}$-Gorenstein local ring $(R, \mathfrak{m})$ coincides with $\operatorname{Ann}_{R}\left(0_{E}^{*}\right)$, the annihilator ideal of the tight closure $0_{E}^{*}$ of the zero submodule in the injective envelope $E=E_{R}(R / \mathfrak{m})$ of the residue field $R / \mathfrak{m}$. Analoguously, for a $d$-dimensional normal local ring $R$, we define the parameter test submodule of the canonical module $\omega_{R}$ by

$$
\tau\left(\omega_{R}\right)=\operatorname{Ann}_{\omega_{R}}\left(0_{H_{\mathrm{m}}^{d}(R)}^{*}\right),
$$

taking into account the duality pairing $\omega_{R} \times H_{\mathfrak{m}}^{d}(R) \rightarrow E$. This notion is a special case of the notion of " $F$-submodule of $\omega_{R}$ " studied by Smith [S1]. Roughly speaking, $\tau\left(\omega_{R}\right)$ (resp. $\left.\tau(R)\right)$ characterizes $F$-rationality (resp. $F$-regularity).

The answer to the first question is reinterpreted in the following theorem.

Theorem 5.2. Let $(R, \mathfrak{m})$ be a normal local ring which is reduced from characteristic zero to characteristic $p \gg 0$, together with a resolution of singularities $f: X \rightarrow \operatorname{Spec} R 1$ Then

$$
\tau\left(\omega_{R}\right)=H^{0}\left(X, \omega_{X}\right) \text { in } \omega_{R} .
$$

Note that $R$ is $F$-rational (resp. $R$ has rational singularities) if and only if $R$ is Cohen-Macaulay and $\tau\left(\omega_{R}\right)=\omega_{R}\left(\right.$ resp. $\left.H^{0}\left(X, \omega_{X}\right)=\omega_{R}\right)$.

The answer to the second question is deduced from the above theorem by using cyclic covering. The main theorem of this paper states that the test ideal $\tau(R)$ has a very geometric expression given by a resolution of singularities.

Theorem 5.9. Let $R$ be a $\mathbb{Q}$-Gorenstein normal ring which is reduced from characteristic zero to characteristic $p \gg 0$, together with a resolution of singularities

\footnotetext{
1 The setup involving "reduction to characteristic $p \gg 0$ " is explained in 4.6, 5.1 and 5.7.
} 
$f: X \rightarrow \operatorname{Spec} R$ whose exceptional set is a simple normal crossing divisor. If we write $K_{X}=f^{*} K_{R}+\Delta$ for an $f$-exceptional $\mathbb{Q}$-divisor $\Delta$, then

$$
\tau(R)=H^{0}\left(X, \mathcal{O}_{X}(\lceil\Delta\rceil)\right) \text { in } R \text {. }
$$

The right-hand side of this equality is called the multiplier ideal ( $\mathrm{E},[\mathrm{N}])$, and plays an important role in birational algebraic geometry. From this theorem we easily reprove the correspondence of $F$-regularity and log terminal singularity, since a $\mathbb{Q}$-Gorenstein local ring $R$ is $F$-regular (resp. log terminal) if and only if $\tau(R)=R$ (resp. $\lceil\Delta\rceil \geq 0$, or equivalently, $H^{0}\left(X, \mathcal{O}_{X}(\lceil\Delta\rceil)\right)=R$ ).

Theorem [5.9] is also proved by Karen E. Smith [S3] in a different way: Smith studies the behavior of the multiplier ideal under the canonical covering quite elegantly, using the log ramification formula. On the other hand, Theorem 5.9 is the most important case of a more general result (Theorem 5.8). In fact, we can prove Theorems 5.8 and 5.9 directly without referring to cyclic covering. The alternative proof, being a little bit laborious, is achieved along the same line as in the proof of Theorem 5.2 The entire proof of this alternative method is not recorded in this paper, but its underlying spirit is found in the proof of Theorem 6.4.

This paper is organized as follows. In Section 1, we collect some generalities and preliminary results on tight closure and test ideals. Section 2 is devoted to studying the behavior of test ideals under cyclic covering. In Section 3, we define the test submodule $\tau\left(\omega_{R}\right)$ of the canonical module $\omega_{R}$, via which Smith's characterization of the tight closure $0_{H_{\mathrm{m}}^{d}(R)}^{*}$ is restated. After reviewing the correspondence of " $F$ rings" and singularities in characteristic zero in terms of reduction modulo $p$ in Section 4, we proceed to Section 5, where the main results of this paper are proved. We also give some "dual forms" of Theorems 5.2 and 5.9 in this section. In Section 6 , we treat the case of graded rings, and give the "graded version" of the above theorems.

Acknowledgements. A part of this work was done at the University of Michigan, where the author stayed in the fall of 1996. The author expresses his deep gratitude to Professor Melvin Hochster and especially to Professor Karen E. Smith for their hospitality and interest to the subject. He also thanks Professor Smith for sending the paper [S3. Thanks are also due to Professor Kei-ichi Watanabe, who had many discussions with the author and gave him helpful comments.

\section{Tight Closure And test ideals}

We first review some generalities about tight closure and test ideals. The reader is referred to Hochster and Huneke [HH1, $\mathrm{HH} 2, \mathrm{HH} 3]$ and Huneke $\mathrm{Hu}$ ] for more detail.

Throughout this paper all rings are excellent commutative rings with unity. For a ring $R, R^{\circ}$ will denote the set of elements of $R$ which are not in any minimal prime ideal. We will often work over a field of characteristic $p>0$. In this case we always use the letter $q$ for a power $p^{e}$ of $p$. Also, for an ideal $I$ of $R, I^{[q]}$ will denote the ideal of $R$ generated by the $q$ th powers of elements of $I$. The ring $R$ viewed as an $R$-module via the $e$-times iterated Frobenius map $F^{e}: R \rightarrow R$ is denoted by ${ }^{e} R$. If $R$ is reduced, then $F^{e}: R \rightarrow{ }^{e} R$ is identified with the natural inclusion map $R \hookrightarrow R^{1 / q}$.

Definition 1.1 ([HH1]). Let $R$ be a Noetherian ring of characteristic $p>0$, and let $I \subset R$ be an ideal. The tight closure $I^{*}$ of $I$ in $R$ is the ideal defined by $x \in I^{*}$ 
if and only if there exists $c \in R^{\circ}$ such that $c x^{q} \in I^{[q]}$ for all $q=p^{e} \gg 0$. We say that $I$ is tightly closed if $I^{*}=I$.

\subsection{Basic Properties ([HH1]).}

(i) If $R$ is regular, then all ideals of $R$ are tightly closed.

(ii) Colon Capturing. Let $R$ be a locally equidimensional Noetherian ring that is a homomorphic image of a Cohen-Macaulay ring, and let $x_{1}, \ldots, x_{n} \in R$ be "parameters," i.e., any $i$ of them $(1 \leq i \leq n)$ generate an ideal of height $i$. Then

$$
\left(x_{1}, \ldots, x_{n-1}\right):_{R} x_{n} \subseteq\left(x_{1}, \ldots, x_{n-1}\right)^{*} .
$$

(iii) For any ideal $I$ of $R$, one has $I^{*} \subseteq \bar{I}$, where $\bar{I}$ denotes the integral closure of the ideal $I \subseteq R$.

Definition 1.3 ([HH1], [FW]). Let $R$ denote a Noetherian ring of characteristic $p>0$.

(i) $\quad R$ is said to be weakly F-regular if every ideal of $R$ is tightly closed. We say that $R$ is F-regular if every localization of $R$ is weakly $F$-regular.

(ii) A local ring $(R, \mathfrak{m})$ is said to be $F$-rational if some (or, equivalently, every) ideal generated by a system of parameters of $R$ is tightly closed. When $R$ is not local, we say that $R$ is $F$-rational if every local ring of $R$ is $F$-rational.

1.4. Remark. It immediately follows from the definition and 1.2 that

regular $\Rightarrow F$-regular $\Rightarrow F$-rational $\Rightarrow$ Cohen-Macaulay and normal.

Also, a Gorenstein $F$-rational ring is $F$-regular.

1.5. Definition and discussion. For an $R$-submodule $N \subseteq M$, we can define the tight closure $N_{M}^{*}$ of $N$ in $M$ as well [HH1]. In the sequel we only treat the case $N=0$, and define the tight closure $0_{M}^{*} \subseteq M$ of the zero submodule in $M$. Let $F^{e}: M=R \otimes_{R} M \rightarrow{ }^{e} R \otimes_{R} M$ be the $e$-times iterated Frobenius map induced on an $R$-module $M$ sending $z \in M$ to $z^{q}:=F^{e}(z)=1 \otimes z \in{ }^{e} R \otimes_{R} M$. Then $0_{M}^{*} \subseteq M$ is defined by

$z \in 0_{M}^{*} \Longleftrightarrow$ there exists $c \in R^{\circ}$ such that $c z^{q}:=c \otimes z=0$ for all $q=p^{e} \gg 0$.

Note that $c \in R$ acts on ${ }^{e} R \otimes_{R} M$ from the left via the identification $R={ }^{e} R$.

If $I \subseteq R$ is an ideal, then $I^{*} / I \cong 0_{R / I}^{*}$. Moreover, the finitistic tight closure $0_{M}^{* f g} \subseteq M$ of the zero submodule in $M$ is defined by

$$
0_{M}^{* f g}:=\bigcup_{M^{\prime}} 0_{M^{\prime}}^{*}
$$

where $M^{\prime}$ runs through all finitely generated $R$-submodules of $M$. One has $0_{M}^{* f g} \subseteq$ $0_{M}^{*}$ in general, and if $M$ itself is finitely generated, then $0_{M}^{* f g}=0_{M}^{*}$.

The important case for us is that $(R, \mathfrak{m})$ is a $d$-dimensional local ring and $M=$ $H_{\mathfrak{m}}^{d}(R)$ or $E_{R}(R / \mathfrak{m})$, the injective hull of the residue field. In the former case, for a system of parameters $x_{1}, \ldots, x_{d}$ of $R$ we have

$$
H_{\mathfrak{m}}^{d}(R) \cong \lim _{\longrightarrow} R /\left(x_{1}^{t}, \ldots, x_{d}^{t}\right),
$$

so that ${ }^{e} R \otimes_{R} H_{\mathfrak{m}}^{d}(R) \cong \underline{\lim R} R /\left(x_{1}^{q t}, \ldots, x_{d}^{q t}\right) \cong H_{\mathfrak{m}}^{d}(R)$ as groups. Thus we see that $(R, \mathfrak{m})$ is $F$-rational if and only if it is Cohen-Macaulay and $0_{H_{\mathfrak{m}}^{d}(R)}^{*}=0$. 
Definition 1.6 ([HH1, $\mathrm{HH} 3]$ ). Let $R$ denote a Noetherian ring of characteristic $p>0$.

(i) The test ideal $\tau(R)$ of $R$ is defined by $\tau(R):=\bigcap_{M} \operatorname{Ann}_{R}\left(0_{M}^{*}\right)$, where the intersection is taken over all finitely generated $R$-modules $M$.

(ii) When $(R, \mathfrak{m})$ is a local ring, the parameter test ideal $\tau_{\text {par }}(R)$ of $R$ is defined by $\tau_{\text {par }}(R):=\bigcap_{I}\left(I:_{R} I^{*}\right)$, where $I$ runs through all ideals generated by a system of parameters of $R$.

An element of $\tau(R) \cap R^{\circ}$ (resp. $\tau_{\text {par }}(R) \cap R^{\circ}$ ) is called a test element (resp. parameter test element) of $R$.

1.7. Remark. (i) It is easy to see that $R$ is weakly $F$-regular if and only if $\tau(R)=$ $R$, and a local ring $(R, \mathfrak{m})$ is $F$-rational if and only if it is Cohen-Macaulay and $\tau_{\text {par }}(R)=R$.

(ii) An element $c \in R^{\circ}$ is a test element (resp. parameter test element) if and only if, for every ideal (resp. every parameter ideal) $I \subset R$ and every $x \in R$, one has

$$
x \in I^{*} \Longleftrightarrow c x^{q} \in I^{[q]} \text { for all } q=p^{0}, p^{1}, p^{2}, \ldots .
$$

The name "test element" comes from this property.

Let $R$ be a normal ring with a canonical module $\omega_{R}$. We say that $R$ is $\mathbb{Q}$ Gorenstein if there is a positive integer $r$ such that the reflexive hull $\omega_{R}^{(r)}$ of the $R$-module $\omega_{R}^{\otimes r} /$ torsion is a locally free $R$-module (in other words, $r K_{R}$ is a Cartier divisor on $\operatorname{Spec} R$, where $K_{R}$ denotes a canonical divisor of $\operatorname{Spec} R$ ).

Theorem 1.8. Let $R$ be a Noetherian ring of characteristic $p>0$ and let $E=$ $\bigoplus_{\mathfrak{m}} E_{R}(R / \mathfrak{m})$, where $\mathfrak{m}$ runs through all maximal ideals of $R$. Then:

(i) $($ HH1] $) \quad \tau(R)=\bigcap_{\mathfrak{m}} \operatorname{Ann}_{R_{\mathfrak{m}}}\left(0_{E_{R}(R / \mathfrak{m})}^{* f g}\right)=\operatorname{Ann}_{R}\left(0_{E}^{* f g}\right)$, where the intersection in the middle term is taken over all maximal ideals of $R$.

(ii) $(\mathrm{AM}],[\mathrm{Mc},[\mathrm{S3}])$ If $R$ is normal and $\mathbb{Q}$-Gorenstein, $\tau(R)=\operatorname{Ann}_{R}\left(0_{E}^{*}\right)$.

(iii) ([HH3], [S1]) If $(R, \mathfrak{m})$ is a d-dimensional Cohen-Macaulay local ring, then $\tau_{\mathrm{par}}(R)=\operatorname{Ann}_{R}\left(0_{H_{\mathrm{m}}^{d}(R)}^{*}\right)$. In particular, if $R$ is Gorenstein, then $\tau(R)=\tau_{\mathrm{par}}(R)$.

To deduce Theorem 1.8 (ii) from (i), one has to prove the equality $0_{E}^{*}=0_{E}^{* f g}$. This is essentially achieved in $[\mathrm{AM}]$ and $[\mathrm{Mc}$ by using the " $F$-bounded" property introduced by Williams [Wi]. In fact, we want to use this result under a slightly different hypothesis. (We assume normality instead of the Cohen-Macaulay property.) So, in the appendix, we give a proof to the following theorem according to the method of [Mc] and [Wi]. This is also proved in [S3] under the same hypothesis as ours.

Theorem 1.9. Let $(R, \mathfrak{m})$ be a d-dimensional excellent normal local ring of characteristic $p>0$ and let $J \subseteq R$ be a divisorial ideal such that the divisor class $\operatorname{cl}(J) \in \mathrm{Cl}(R)$ has a finite order. Then $0_{H_{\mathrm{m}}^{d}(J)}^{*}=0_{H_{\mathfrak{m}}^{d}(J)}^{* g}$.

Definition 1.10 ([H]2]). Let $R$ be a reduced ring of characteristic $p>0$ which is $F$-finite (i.e., the Frobenius map $F: R \rightarrow{ }^{1} R$ is finite). We say that $R$ is strongly $F$-regular if, for any $c \in R^{\circ}$ there exists a power $q=p^{e}$ such that the inclusion map $c^{1 / q} R \hookrightarrow R^{1 / q}$ splits as an $R$-linear map.

1.11. Remark. For an $F$-finite reduced $\operatorname{ring} R$, we have the implications "strongly $F$-regular $\Rightarrow F$-regular $\Rightarrow$ weakly $F$-regular," and $R$ is strongly $F$-regular if and 
only if $0_{E}^{*}=0$, where $E=\bigoplus_{\mathfrak{m}} E_{R}(R / \mathfrak{m})$ as in 1.8, In particular, for a $\mathbb{Q}$-Gorenstein ring the three variations of $F$-regularity are all the same ([Mc], see also [LS1], Wi] $)$. More generally, the equality $0_{E}^{*}=0_{E}^{* f g}$ implies that the test ideal commutes with localization and that the test ideal is a defining ideal of the non- $F$-regular locus, see the recent result of Lyubeznik and Smith [LS2].

Theorem 1.12 ([HH1], [HH2]). Let $R$ be a reduced ring of characteristic $p>0$ and let $R_{c}$ be the localization at an element $c \in R^{\circ}$.

(i) If $R$ is essentially of finite type over an excellent local ring and $R_{c}$ is regular, then some power $c^{n}$ of $c$ is a test element of $R$.

(ii) If $R$ is $F$-finite and $R_{c}$ is strongly $F$-regular, then some power $c^{n}$ of $c$ is a test element of $R$.

\section{The BehaVIOR OF TEST IDEALS UNDER CYCLIC COVERING}

In this section, we investigate the behavior of test ideals under cyclic covering, based on an idea due to K.-i. Watanabe. The author thanks Watanabe for informing him of the result proved in [NW], which is not published yet.

2.1. Cyclic covering. Let $R$ be a normal domain and $I \subset R$ a divisorial ideal. For an integer $i$, we denote by $I^{(i)}$ the reflexive hull of $I^{i}$. We assume that the class $\operatorname{cl}(I)$ of $I$ in the divisor class group $\mathrm{Cl}(R)$ has finite order $r>0$, i.e., $I^{(r)}=v R$ for some $v \in R$. For a multiple $n \in \mathbb{N}$ of $r$ we fix a $w \in R$ such that $I^{(n)}=w R$, and consider an $R$-algebra $S=\left(\bigoplus_{i \geq 0} I^{(i)} z^{i}\right) /\left(w z^{n}-1\right)$, where $z$ is an indeterminate of degree 1 . We denote the image of $z$ in $S$ by $u$. Then $S=\bigoplus_{i=0}^{n-1} I^{(i)} u^{i}$ has a natural $\mathbb{Z}_{n}=\mathbb{Z} / n \mathbb{Z}$-graded $R$-algebra structure. $S$ is called a cyclic cover of $R$. In particular, if $I$ is isomorphic to the canonical module $\omega_{R}$ of $R, S$ is called a canonical cover of $R$. See [TW] for a more detailed ring-theoretic study of $S$ (the normality of $S$, etc.).

2.2. Discussion. With the notation as in 2.1 , assume that $(R, \mathfrak{m})$ is a local ring of dimension $d$. Then $S$ is a semilocal ring, since $R \rightarrow S$ is finite. Let $\mathfrak{n}_{1}, \ldots, \mathfrak{n}_{s}$ be the maximal ideals of $S$. In the sequel we frequently use the following easy fact: For non-negative integers $i, j$ with $i \not \equiv i+j \equiv 0 \bmod r$, one has $I^{(i)} \cdot I^{(j)} \subseteq$ $\mathfrak{m} I^{(i+j)}$. Indeed, if this is not the case, then there exist $x \in I^{(i)}$ and $y \in I^{(j)}$ such that $I^{(i+j)}=x y R=I^{(i)} \cdot I^{(j)}$. But this implies that $x R=y^{-1} I^{(i)} \cdot I^{(j)}=I^{(i)}$, contradicting $i \not \equiv 0 \bmod r$.

Let $\mathfrak{n}$ be the $\mathbb{Z}_{n}$-graded $R$-submodule of $S$ whose graded piece of degree $i$ mod $n(0 \leq i \leq n-1)$ is $\mathfrak{m} I^{(i)} u^{i}$ for $i \equiv 0 \bmod r$, and $I^{(i)} u^{i}$ for $i \not \equiv 0 \bmod r$. Then the above fact tells us that $\mathfrak{n}$ is an ideal of $S$. Even more, $\mathfrak{n}$ is the Jacobson radical of $S$. To see this, we consider an $R$-subalgebra $T=\bigoplus_{j=0}^{t-1} I^{(j r)} u^{j r}$ of $S$, where $t=n / r$. Then $R \hookrightarrow T$ is étale since each $I^{(j r)}$ is principal, so that $\mathfrak{m} T=\bigoplus_{j=0}^{t-1} \mathfrak{m} I^{(j r)} u^{j r}$ is the Jacobson radical of $T$. Hence $S / \mathfrak{n} \cong T / \mathfrak{m} T$ is a zero-dimensional reduced ring, so $\mathfrak{n}$ is a radical ideal containing $\sqrt{\mathfrak{m} S}$. On the other hand, if $x \in S$ is a homogeneous element of degree $\not \equiv 0 \bmod r$, then $x^{r} \in \mathfrak{m} S$ by the fact we observed above, whence $x \in \sqrt{\mathfrak{m} S}$. Thus we conclude that

$$
\mathfrak{n}=\sqrt{\mathfrak{m} S}=\mathfrak{n}_{1} \cap \cdots \cap \mathfrak{n}_{s} .
$$

Now let $E_{R}:=E_{R}(R / \mathfrak{m}) \cong H_{\mathfrak{m}}^{d}\left(\omega_{R}\right), E_{T}:=E_{T}(T / \mathfrak{m} T) \cong H_{\mathfrak{m}}^{d}\left(\omega_{T}\right)$ and $E_{S}:=$ $E_{S}(S / \mathfrak{n})=\bigoplus_{i=1}^{s} E_{S}\left(S / \mathfrak{n}_{i}\right) \cong H_{\mathfrak{m}}^{d}\left(\omega_{S}\right)$. Then it easily follows that the "socles" 
of these are $\left(0:_{E_{R}} \mathfrak{m}\right)=R / \mathfrak{m},\left(0:_{E_{T}} \mathfrak{m} T\right)=T / \mathfrak{m} T$, and $\left(0:_{E_{S}} \mathfrak{n}\right)=S / \mathfrak{n}=$ $\bigoplus_{i=1}^{s} S / \mathfrak{n}_{i}$, respectively. On the other hand, since $R \rightarrow S$ is finite of relative dimension 0 , one has that $\omega_{S} \cong \operatorname{Hom}_{R}\left(S, \omega_{R}\right)=\operatorname{Hom}_{R}\left(\bigoplus_{i=0}^{n-1} I^{(i)} u^{i}, \omega_{R}\right)$ by the adjunction formula. Hence $E_{S} \cong H_{\mathfrak{m}}^{d}\left(\omega_{S}\right)$ has a natural $\mathbb{Z}_{n}$-graded $S$-module structure, and its graded piece of degree $i$ is

$$
\begin{aligned}
{\left[E_{S}\right]_{i} } & \cong H_{\mathfrak{m}}^{d}\left(\operatorname{Hom}_{R}\left(I^{(-i)}, \omega_{R}\right)\right) \\
& \cong \operatorname{Hom}_{R}\left(\operatorname{Hom}_{R}\left(\operatorname{Hom}_{R}\left(I^{(-i)}, \omega_{R}\right), \omega_{R}\right), E_{R}\right)=\operatorname{Hom}_{R}\left(I^{(-i)}, E_{R}\right)
\end{aligned}
$$

by the local dualty. Note that $E_{R}$ (resp. $\left.E_{T} \cong \bigoplus_{j=0}^{t-1} \operatorname{Hom}_{R}\left(I^{(-j r)}, E_{R}\right)\right)$ is embedded in $E_{S}$ as the graded part of degree 0 (resp. degree $\equiv 0 \bmod r$ ).

Lemma 2.3 (cf. $[\mathbf{N W})$. With the notation as in 2.2, $\left(0: E_{S} \mathfrak{n}\right)$ sits in the graded part of $E_{S}$ of degree $\equiv 0 \bmod r$.

Proof. It is sufficient to prove $\left(0:_{E_{T}} \mathfrak{m}\right) \subseteq\left(0: E_{S} \mathfrak{n}\right)$. Indeed, this implies that $\left(0: E_{S} \mathfrak{n}\right)=\left(0: E_{T} \mathfrak{m}\right) \subset E_{T}$, since $\left(0:_{E_{T}} \mathfrak{m}\right)=T / \mathfrak{m} T$ and $\left(0: E_{S} \mathfrak{n}\right)=S / \mathfrak{n}$ are both $R / \mathfrak{m}$-vector spaces of the same dimension $t=n / r$.

Let $\xi \in\left(0:_{E_{T}} \mathfrak{m}\right)$ be a homogeneous element of degree $j r$ in the $\mathbb{Z}_{n}$-grading of $E_{T} \subseteq E_{S}$. To show $\xi \in\left(0: E_{S} \mathfrak{n}\right)$, it suffices to prove that $a u^{i} \xi=0$ for every $a \in I^{(i)}$ with $i \not \equiv 0 \bmod r$. If we regard $\xi$ as an element of $\operatorname{Hom}_{R}\left(I^{(-j r)}, E_{R}\right) u^{j r}$, then $a u^{i} \xi \in \operatorname{Hom}_{R}\left(I^{(-i-j r)}, E_{R}\right) u^{i+j r}$ is defined by $\left(a u^{i} \xi\right)(x)=\xi(a x) u^{i}$ for every $x \in I^{(-i-j r)}$. However, since $a x \in I^{(i)} \cdot I^{(-i-j r)} \subseteq \mathfrak{m} I^{(-j r)}$ as we have seen in 2.2 there exist $b \in \mathfrak{m}$ and $y \in I^{(-j r)}$ such that $a x=b y$, so that $\left(a u^{i} \xi\right)(x)=\xi(b y) u^{i}=$ $b \xi(y) u^{i}=0$. Hence $a u^{i} \xi=0$ as required.

Proposition 2.4. With the notation as in 2.2 assume in addition that $R$ is of characteristic $p>0$ and $n$ is not divisible by $p$. Then $0_{E_{S}}^{*}$ is a $\mathbb{Z}_{n}$-graded $S$ submodule of $E_{S}$, and its graded piece of degree $i$ is $0_{\left[E_{S}\right]_{i}}^{*}$, the tight closure of zero in $\left[E_{S}\right]_{i}$ as an $R$-module. In particular, the graded piece of $0_{E_{S}}^{*}$ of degree $\equiv 0 \bmod n$ is isomorphic to $0_{E_{R}}^{*}$.

Proof. Since $n$ is not divisible by $p$, the inclusion $R \hookrightarrow S$ is étale in codimension 1 , so that the map ${ }^{e} R \otimes_{R} S \rightarrow{ }^{e} S$ is isomorphic in codimension 1 by [W2]. This implies that ${ }^{e} R \otimes_{R} H_{\mathfrak{m}}^{d}\left(\omega_{S}\right) \cong{ }^{e} S \otimes_{S} H_{\mathfrak{m}}^{d}\left(\omega_{S}\right)$ and that the $e$-times Frobenius map on $E_{S} \cong H_{\mathfrak{m}}^{d}\left(\omega_{S}\right)$ as $S$-module can be identified with that as $R$-module, $F^{e}: E_{S} \rightarrow$ ${ }^{e} R \otimes_{R} E_{S}=\bigoplus_{i=0}^{n-1} e^{e} R \otimes_{R}\left[E_{S}\right]_{i}$. (See the proof of [W2, Theorem 2.7].) Now let $\xi \in 0_{E_{S}}^{*}$ and write $\xi=\xi_{0}+\xi_{1}+\cdots+\xi_{n-1}$ with $\xi_{i} \in\left[E_{S}\right]_{i}$. Then there exists a $c \in S^{\circ}$ such that $c \xi^{q}=0$ in ${ }^{e} S \otimes_{S} E_{S} \cong{ }^{e} R \otimes_{R} E_{S}$. As $R \hookrightarrow S$ is finite, there is a $b \in R^{\circ} \cap c S$ with $b \xi^{q}=\sum_{i=0}^{n-1} b \xi_{i}^{q}=0$. This implies $b \xi_{i}^{q}=0$ for $0 \leq i \leq n-1$, since $b \xi_{0}^{q}, \ldots, b \xi_{n-1}^{q}$ are in different graded pieces of ${ }^{e} R \otimes_{R} E_{S}=\bigoplus_{i=0}^{n-1} e_{R} \otimes_{R}\left[E_{S}\right]_{i}$. Hence $\xi_{i} \in 0_{\left[E_{S}\right]_{i}}^{*} \subseteq 0_{E_{S}}^{*}$ for each $i$, whence $0_{E_{S}}^{*}$ is graded. It is now obvious that $\left[0_{E_{S}}^{*}\right]_{i}=0_{\left[E_{S}\right]_{i}}^{*}$.

Theorem 2.5. With the notation as in 2.2, assume in addition that $R$ is of characteristic $p>0$ and $n$ is not divisible by $p$. Then $\operatorname{Ann}_{S}\left(0_{E_{S}}^{*}\right)$ is a homogeneous ideal in the $\mathbb{Z}_{n}$-grading of $S$, and its graded piece of degree 0 is

$$
\operatorname{Ann}_{R}\left(0_{E_{R}}^{*}\right)=\operatorname{Ann}_{S}\left(0_{E_{S}}^{*}\right) \cap R .
$$

Proof. By Proposition 2.4 it is obvious that $\operatorname{Ann}_{R}\left(0_{E_{R}}^{*}\right) \supseteq \operatorname{Ann}_{S}\left(0_{E_{S}}^{*}\right) \cap R$. For the converse, assume to the contrary that there exists $c \in \operatorname{Ann}_{R}\left(0_{E_{R}}^{*}\right) \backslash \operatorname{Ann}_{S}\left(0_{E_{S}}^{*}\right)$. 
Then there is a $\xi \in 0_{E_{S}}^{*}$ such that $c \xi \neq 0$. We may assume that $\xi$ is homogeneous in the $\mathbb{Z}_{n}$-grading of $E_{S}$. Pick a homogeneous element $b \in S$ such that $b(c \xi)$ is a nonzero element of the socle $\left(0: E_{S} \mathfrak{n}\right)$ of $E_{S}$, which sits in degree $\equiv 0 \bmod r$ by Lemma 2.3. Since the degree of $c \in R$ is 0 , the degree of $b \xi$ is also divisible by $r$, say $\operatorname{deg}(b \xi)=j r$. Then we have $b \xi \in\left[0_{E_{S}}^{*}\right]_{j r} \cong 0_{E_{R}}^{*}$ by Proposition 2.4. It follows that $b(c \xi)=c(b \xi)=0$, a contradiction.

Corollary 2.6. Let $R$ be a $\mathbb{Q}$-Gorenstein normal domain of characteristic $p>0$, and let $I \subseteq R$ be a divisorial ideal such that $I^{(n)} \cong R$ for some $n \in \mathbb{N}$ which is not divisible by $p$. Let $S=\bigoplus_{i=0}^{n-1} I^{(i)} u^{i}$ be a cyclic covering of $R$. Then $\tau(R)=\tau(S) \cap R$.

Proof. For any maximal ideal $\mathfrak{m}$ of $R$, we have $\tau(R)_{\mathfrak{m}}=\tau\left(R_{\mathfrak{m}}\right)$ and $(\tau(S) \cap R)_{\mathfrak{m}}=$ $\tau\left(S_{\mathfrak{m}}\right) \cap R_{\mathfrak{m}}$ by Theorem 1.8 (i), since taking annihilator and intersection are preserved under localization. Hence we may assume that $R$ is local. Note also that the $\mathbb{Q}$-Gorensteinness of $R$ implies that $S$ is $\mathbb{Q}$-Gorenstein, because $R \rightarrow S$ is étale in codimension 1. Therefore the result follows from the above theorem and Theorem 1.8 (ii).

\section{The PARAmeter test module}

The test ideal $\tau(R)$ of a ring $R$ measures how far the ring is from $F$-regular. Similarly, the parameter test ideal is a measure of the distance from $F$-rational. However, if we observe Theorem 1.8 from the viewpoint of the Matlis duality, we find that the test ideal has an advantage over the parameter test ideal, because $\tau(R)$ and $0_{E}^{* f g}$ respectively lie in $R$ and its Matlis dual $E$, while this is not the case for the parameter test ideal in general. This simple observation suggests that it is more natural to consider the following notion instead of the parameter test ideal (see also [S1]).

Definition 3.1. Let $(R, \mathfrak{m})$ be a $d$-dimensional Noetherian normal local ring of characteristic $p>0$. We define the parameter test submodule $\tau\left(\omega_{R}\right)$ of the canonical module $\omega_{R}$ of $R$ by

$$
\tau\left(\omega_{R}\right)=\operatorname{Ann}_{\omega_{R}}\left(0_{H_{\mathrm{m}}^{d}(R)}^{*}\right),
$$

the annihilator of $0_{H_{\mathfrak{m}}^{d}(R)}^{*}$ in $\omega_{R}$ with respect to the duality pairing $\omega_{R} \times H_{\mathfrak{m}}^{d}(R) \rightarrow$ $E=E_{R}(R / \mathfrak{m})$.

3.2. Remark. (i) If $(R, \mathfrak{m})$ is quasi-Gorenstein (i.e., $R \cong \omega_{R}$, with $R$ possibly being non-Cohen-Macaulay), then $\tau(R)$ and $\tau\left(\omega_{R}\right)$ coincide with each other via $R \cong \omega_{R}$, by Theorem 1.8, Also, if $(R, \mathfrak{m})$ is Gorenstein, we can identify $\tau(R), \tau_{\text {par }}(R)$ and $\tau\left(\omega_{R}\right)$ via $R \cong \omega_{R}$.

(ii) We can extend the above definition also to the case when $R$ is not local as in 1.8. Namely, for a normal ring $R$ with a canonical module $\omega_{R}, \tau\left(\omega_{R}\right)$ is defined to be the unique $R$-submodule $\bigcap_{\mathfrak{m}} \tau\left(\omega_{R_{\mathrm{m}}}\right)$ of $\omega_{R}$, where $\mathfrak{m}$ runs through all maximal ideals of $R$. It is easy to check that $\tau\left(\omega_{R}\right)_{\mathfrak{m}}=\tau\left(\omega_{R_{\mathfrak{m}}}\right)$ for every maximal ideal $\mathfrak{m}$.

For later use we state the following easy lemma, the proof of which needs only some elementary facts about the Matlis duality (cf. [S1, Lemma 2.1]).

Lemma 3.3. Let $M$ be a finitely generated module over a Noetherian local ring $(R, \mathfrak{m})$, and denote its Matlis dual by $M^{\prime}=\operatorname{Hom}_{R}\left(M, E_{R}(R / \mathfrak{m})\right)$. Let $N$ (resp. L) be any submodule of $M$ (resp. $\left.M^{\prime}\right)$. If $L=\operatorname{Ann}_{M^{\prime}} N$, then $N=\operatorname{Ann}_{M} L$. If $(R, \mathfrak{m})$ is complete, the converse also holds true. 
Now we recall the following interesting characterization of the tight closure $0_{H_{\mathrm{m}}^{d}(R)}^{*}$ due to Karen Smith according to a generalization by herself.

Theorem 3.4 (K. E. Smith [S2]). Let $(R, \mathfrak{m})$ be a d-dimensional excellent local ring of characteristic $p>0$. Then the tight closure of zero in $H_{\mathfrak{m}}^{d}(R), 0_{H_{\mathfrak{m}}^{d}(R)}^{*}$, is the maximal proper $R$-submodule of $H_{\mathfrak{m}}^{d}(R)$ which is stable under the Frobenius action on $H_{\mathfrak{m}}^{d}(R)$.

Corollary 3.5. Let $(R, \mathfrak{m})$ be a normal local ring as in 3.4 and let $f: X \rightarrow \operatorname{Spec} R$ be a proper birational morphism from a normal scheme $X$. Then one has a natural inclusion $\operatorname{Ann}_{H_{\mathrm{m}}^{d}(R)} H^{0}\left(X, \omega_{X}\right) \subseteq 0_{H_{\mathrm{m}}^{d}(R)}^{*}$, or dually, $\tau\left(\omega_{R}\right) \subseteq H^{0}\left(X, \omega_{X}\right)$ in $\omega_{R}$.

Proof. Let $Z=f^{-1}(\mathfrak{m})$ be the closed fiber of $f$. By [S2, Proposition 1.12] we have a commutative diagram

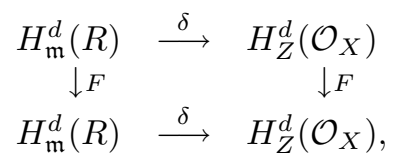

where $F$ denotes the Frobenius map on $H_{\mathfrak{m}}^{d}(R)$ (resp. $H_{Z}^{d}\left(\mathcal{O}_{X}\right)$ ), and $\delta$ is an edge map of the Leray spectral sequence $H_{\mathfrak{m}}^{i}\left(H^{j}\left(X, \mathcal{O}_{X}\right)\right) \Longrightarrow H_{Z}^{i+j}\left(\mathcal{O}_{X}\right)$, which is the Matlis dual of the natural inclusion map $H^{0}\left(X, \omega_{X}\right) \rightarrow \omega_{R}$. (See the careful treatments in [LT], [S2].) Since $\omega_{R} / H^{0}\left(X, \omega_{X}\right)$ is a torsion module, $\operatorname{Ker}(\delta)$ is a proper submodule of $H_{\mathfrak{m}}^{d}(R)$ which is stable under the Frobenius action on $H_{\mathfrak{m}}^{d}(R)$. Hence

$$
\operatorname{Ker}(\delta)=\operatorname{Hom}_{R}\left(\omega_{R} / H^{0}\left(X, \omega_{X}\right), E\right)=\operatorname{Ann}_{H_{\mathfrak{m}}^{d}(R)} H^{0}\left(X, \omega_{X}\right)
$$

is contained in $0_{H_{\mathfrak{m}}^{d}(R)}^{*}$ by Theorem 3.4 The dual form $H^{0}\left(X, \omega_{X}\right) \supseteq \tau\left(\omega_{R}\right)$ follows from Lemma 3.3 .

3.6. Cyclic covering revisited. Let $(R, \mathfrak{m})$ be a $d$-dimensional normal local ring, let $I \subseteq R$ be a divisorial ideal with $I^{(n)} \cong R$ for some $n \in \mathbb{N}$, and let $S=\bigoplus_{i=0}^{n-1} I^{(i)}$ be a cyclic cover of $R$. Let $E_{R}=E_{R}(R / \mathfrak{m})$ and $E_{S}=E_{S}(S / \mathfrak{n})$ be as in 2.2 Then $H_{\mathfrak{n}}^{d}(S)=\bigoplus_{i=0}^{n-1} H_{\mathfrak{m}}^{d}\left(I^{(i)}\right)$ and $\omega_{S}=\bigoplus_{i=0}^{n-1} \operatorname{Hom}_{R}\left(I^{(-i)}, \omega_{R}\right)$ as $\mathbb{Z}_{n}$-graded $S$-modules.

Proposition 3.7. With the notation as above, assume in addition that $R$ is of characteristic $p>0$ and $n$ is not divisible by $p$. Then:

(i) $0_{H_{\mathfrak{n}}^{d}(S)}^{*}$ is a $\mathbb{Z}_{n}$-graded $S$-submodule of $H_{\mathfrak{n}}^{d}(S)$, and its degree $i$ part is

$$
\left[0_{H_{\mathfrak{n}}^{d}(S)}^{*}\right]_{i}=0_{H_{\mathfrak{m}}^{d}\left(I^{(i)}\right)}^{*} \quad \text { in }\left[H_{\mathfrak{n}}^{d}(S)\right]_{i}=H_{\mathfrak{m}}^{d}\left(I^{(i)}\right) .
$$

(ii) $\tau\left(\omega_{S}\right)$ is a $\mathbb{Z}_{n}$-graded $S$-submodule of $\omega_{S}$, and its degree $i$ part is

$$
\left[\tau\left(\omega_{S}\right)\right]_{i}=\operatorname{Ann}_{\operatorname{Hom}_{R}\left(I^{(-i)}, \omega_{R}\right)}\left(0_{H_{\mathrm{m}}^{d}\left(I^{(i)}\right)}^{*}\right) \quad \text { in }\left[\omega_{S}\right]_{i}=\operatorname{Hom}_{R}\left(I^{(-i)}, \omega_{R}\right) .
$$

Proof. The proof is quite similar to the proofs of 2.4 and 2.5 if one notes that the duality pairings $\omega_{S} \times H_{\mathfrak{n}}^{d}(S) \rightarrow E_{S}$ as $S$-modules and $\operatorname{Hom}_{R}\left(I^{(-i)}, \omega_{R}\right) \times H_{\mathfrak{m}}^{d}\left(I^{(i)}\right) \rightarrow$ $E_{R}$ as $R$-modules are compatible.

\section{Singularities in characteristic zero and Reduction modulo $p$}

Although tight closure is at first glance a characteristic $p$ notion, it turns out to characterize singularities of algebraic varieties in characteristic zero. First of all we shall recall some conventions and definitions. For a $\mathbb{Q}$-divisor $D$ on a normal 
variety $Y$, we denote its round-up and round-down by $\lceil D\rceil$ and $\lfloor D\rfloor$, respectively. We sometimes denote the divisorial sheaf $\mathcal{O}_{Y}(\lfloor D\rfloor)$ simply by $\mathcal{O}_{Y}(D)$.

Definition 4.1. Let $Y$ be a normal variety over a field of characteristic zero and let $f: X \rightarrow Y$ be a resolution of singularities whose exceptional set is a simple normal crossing divisor with irreducible components $E_{1}, \ldots, E_{r}$.

(i) $Y$ is said to have rational singularities if $R^{i} f_{*} \mathcal{O}_{X}=0$ for all $i>0$.

(ii) If $Y$ is $\mathbb{Q}$-Gorenstein (i.e., the canonical divisor $K_{Y}$ of $Y$ is $\mathbb{Q}$-Cartier), then one can write the canonical divisor of $X$ as $K_{X}=f^{*} K_{Y}+\Delta$, where $\Delta=\sum_{i=1}^{r} a_{i} E_{i}$ for some $a_{1}, \ldots, a_{r} \in \mathbb{Q}$. We call this $\mathbb{Q}$-divisor $\Delta$ the discrepancy of $f$.

(iii) $Y$ is said to have log terminal singularities if $Y$ is $\mathbb{Q}$-Gorenstein and $a_{i}>-1$ for each $i=1, \ldots, r$, where $\Delta=\sum_{i=1}^{r} a_{i} E_{i}$ is the discrepancy of $f$ as in (ii).

4.2. Remark. Properties (i) and (iii) in 4.1] do not depend on the choice of $f: X \rightarrow$ $Y$; nor does the ideal sheaf $f_{*} \mathcal{O}_{X}(\lceil\Delta\rceil) \subseteq \mathcal{O}_{Y}$ in the situation of (ii). This ideal sheaf is called the multiplier ideal and plays an important role in birational algebraic geometry (see $[\mathrm{E}]$ ).

To relate the above notions with $F$-rational and $F$-regular rings, we use the technique of "reduction modulo $p . "$

Definition 4.3 (cf. $[\mathrm{HR}$ ). Let $R$ be a finitely generated algebra over a field $k$ of characteristic zero. We say that $R$ has $F$-rational type (resp. F-regular type) if there exist a finitely generated $\mathbb{Z}$-subalgebra $A$ of $k$ and a finitely generated $A$-algebra $R_{A}$ satisfying the following conditions:

(i) $R_{A}$ is flat over $A$ and $R_{A} \otimes_{A} k \cong R$.

(ii) $R_{\kappa}=R_{A} \otimes_{A} \kappa(s)$ is $F$-rational (resp. $F$-regular) for every closed point $s$ in a dense open subset of $S=\operatorname{Spec} A$, where $\kappa=\kappa(s)$ denotes the residue field of $s \in S$.

4.4. Remark. In condition (ii), as $A$ is finitely generated over $\mathbb{Z}, \kappa=\kappa(s)$ is a finite field, and so it is a perfect field of positive characteristic. We frequently abbreviate the statement in condition (ii) as "the fiber ring $R_{\kappa}$ is $F$-rational (resp. $F$-regular) for general closed points $s \in S$."

The starting point of the present paper is the following characterization of "mild" singularities via tight closure.

Theorem 4.5 ([Ha, [MS], [S2], [W3]). Let $R$ be a finitely generated algebra over a field of characteristic zero. Then Spec $R$ has rational singularities (resp. log terminal singularities) if and only if $R$ is of F-rational type (resp. of F-regular type and $\mathbb{Q}$ Gorenstein).

To generalize the above theorem to wider classes of singularities, we have to begin with a ring $R$ in characteristic zero, and reduce it to characteristic $p \gg 0$, together with a resolution of singularities $f: X \rightarrow \operatorname{Spec} R$. To see the properties shared by such "general" modulo $p$ reductions, we briefly review the argument in [Ha, 4.2-4.5].

4.6. Discussion. Let $R$ be a normal domain of dimension at least 2 which is finitely generated over a field $k$ of characteristic zero. Let $f: X \rightarrow \operatorname{Spec} R$ be a resolution of singularities whose exceptional set $E \subset X$ is a simple normal crossing divisor, and $\Gamma$ an effective $f$-exceptional divisor such that $-\Gamma$ is $f$-ample. We choose a rational number $\varepsilon>0$ such that the $\mathbb{Q}$-divisor $\varepsilon \Gamma$ has no integral part, and set $D=-\varepsilon \Gamma$. 
Choosing a suitable finitely generated $\mathbb{Z}$-subalgebra $A$ of $k$, one can construct a finitely generated flat $A$-algebra $R_{A}$, a smooth $A$-scheme $X_{A}$ and a birational $A$ morphism $f_{A}: X_{A} \rightarrow \operatorname{Spec} R_{A}$ whose exceptional set $E_{A} \subset X_{A}$ is a simple normal crossing divisor over $A$, such that by tensoring $k$ over $A$ one gets back $R, X$, and $f: X \rightarrow \operatorname{Spec} R$. We also have an $f_{A^{-}}$exceptional divisor $\Gamma_{A}$ which tensors back to $\Gamma$ such that $D_{A}=-\varepsilon \Gamma_{A}$ is an $f_{A}$-ample $\mathbb{Q}$-Cartier divisor supported on $E_{A}$ with $\left\lfloor-D_{A}\right\rfloor=0$.

Given a closed point $s \in S=\operatorname{Spec} A$ with residue field $\kappa=\kappa(s)$, we denote the corresponding fibers over $s$ by $f_{\kappa}: X_{\kappa} \rightarrow \operatorname{Spec} R_{\kappa}$, etc. Obviously, all of the above-mentioned properties are preserved in every closed fiber, i.e., $X_{\kappa}$ is smooth over $\kappa, E_{\kappa}$ is the simple normal crossing exceptional divisor of $f_{\kappa}$, and $D_{\kappa}=$ $-\varepsilon \Gamma_{\kappa}$ is an $f_{\kappa}$-ample $\mathbb{Q}$-Cartier divisor supported on $E_{\kappa}$ such that $\left\lfloor-D_{\kappa}\right\rfloor=0$. Moreover, not only do the fiber rings $R_{\kappa}$ over general closed points $s \in S$ inherit the properties possessed by the original ring $R$ (e.g. normality, Cohen-Macaulay and $\mathbb{Q}$-Gorenstein properties), but also what is true in characteristic zero (such as the Grauert-Riemenschneider vanishing) holds for general closed fibers.

Now we fix a general closed point $s \in S$ with residue field $\kappa=\kappa(s)$ of sufficiently large characteristic $p$, and work over $\kappa$. We refer to the fibers over $s \in S$ as "reduction modulo $p \gg 0$," and use the phrase "in characteristic $p \gg 0$ " when we look at general closed fibers which are reduced from characteristic zero to characteristic $p \gg 0$ as above. Then we have the following vanishing of cohomologies for all $e \geq 0$ [Ha, 4.3]:

(a) $H^{j}\left(X_{\kappa}, \Omega_{X_{\kappa} / \kappa}^{i}\left(\log E_{\kappa}\right)\left(-E_{\kappa}-\left\lfloor-p^{e} D_{\kappa}\right\rfloor\right)\right)=0 \quad$ for $i+j=\operatorname{dim} X_{\kappa}+1$;

(b) $H^{j}\left(X_{\kappa}, \Omega_{X_{\kappa} / \kappa}^{i}\left(\log E_{\kappa}\right)\left(-E_{\kappa}-\left\lfloor-p^{e+1} D_{\kappa}\right\rfloor\right)\right)=0 \quad$ for $i+j=\operatorname{dim} X_{\kappa}$ and $j>0$.

To be brief, vanishing (a) comes from the Akizuki-Nakano vanishing theorem in characteristic zero (cf. Ha, Corollary 3.8]), and (b) is due to the Serre vanishing since $D_{\kappa}$ is ample and $p \gg 0$. Applying [Ha, Proposition 3.6] to (a) and (b), we see that the map $F^{\vee}: H^{0}\left(X_{\kappa}, \mathcal{H o m}_{\mathcal{O}_{X_{\kappa}}}\left(F_{*} \mathcal{O}_{X_{\kappa}}\left(-p^{e+1} D_{\kappa}\right), \omega_{X_{\kappa}}\right)\right) \rightarrow$ $H^{0}\left(X_{\kappa}, \mathcal{H o m}_{\mathcal{O}_{X_{\kappa}}}\left(\mathcal{O}_{X_{\kappa}}\left(-p^{e} D_{\kappa}\right), \omega_{X_{\kappa}}\right)\right)$ induced by the canonical dual of the Frobenius $F: \mathcal{O}_{X_{\kappa}}\left(-p^{e} D_{\kappa}\right) \rightarrow F_{*} \mathcal{O}_{X_{\kappa}}\left(-p^{e+1} D_{\kappa}\right)$ is surjective for all $e \geq 0$. Hence the composition map

$$
\left(F^{e}\right)^{\vee}: H^{0}\left(X_{\kappa}, \mathcal{H o m}_{\mathcal{O}_{X_{\kappa}}}\left(F_{*}^{e} \mathcal{O}_{X_{\kappa}}\left(-q D_{\kappa}\right), \omega_{X_{\kappa}}\right)\right) \rightarrow H^{0}\left(X_{\kappa}, \mathcal{H o m}_{\mathcal{O}_{X_{\kappa}}}\left(\mathcal{O}_{X_{\kappa}}, \omega_{X_{\kappa}}\right)\right)
$$

is surjective for all $q=p^{e}$. Note that the surjectivity of this map implies the surjectivity of the corresponding map after moving to the local ring $\left(R_{\kappa}\right)_{P}$ at every prime ideal $P \in \operatorname{Spec} R_{\kappa}$ and its induced resolution $\left(f_{\kappa}\right)_{P}:\left(X_{\kappa}\right)_{P} \rightarrow \operatorname{Spec}\left(R_{\kappa}\right)_{P}$.

\section{Geometric interpretation of test ideals AND test modules}

To state the results we change the notaiton from 4.6, and put the following:

5.1. Setup. Let $(R, \mathfrak{m})$ be a normal local ring of dimension $d \geq 2$, essentially of finite type over a perfect field $\kappa$ of characteristic $p>0$. Let $f: X \rightarrow$ Spec $R$ be a resolution of singularities with simple normal crossing exceptional divisor $E \subset X$, and let $D$ be an $f$-ample $\mathbb{Q}$-Cartier divisor on $X$ such that $\operatorname{Supp}(D)=E$ and $\lfloor-D\rfloor=0$. We denote the closed fiber of $f$ by $Z$. We assume that $(R, \mathfrak{m})$ is the localization at any prime ideal of a finitely generated $\kappa$-algebra which is a "reduction modulo $p \gg 0$ " as in 4.6, as well as $X, D, E$ and $f: X \rightarrow \operatorname{Spec} R$. This means that the map

$$
\left(F^{e}\right)^{\vee}: H^{0}\left(X, \mathcal{H o m}_{\mathcal{O}_{X}}\left(F_{*}^{e} \mathcal{O}_{X}(-q D), \omega_{X}\right)\right) \rightarrow H^{0}\left(X, \mathcal{H o m}_{\mathcal{O}_{X}}\left(\mathcal{O}_{X}, \omega_{X}\right)\right)
$$


is surjective, or dually, the $e$-times iterated Frobenius map

$$
F^{e}: H_{Z}^{d}\left(\mathcal{O}_{X}\right) \rightarrow H_{Z}^{d}\left(F_{*}^{e} \mathcal{O}_{X}(-q D)\right)
$$

is injective for every $q=p^{e}$.

The following theorem is obtained independently in [MS].

Theorem 5.2. Let the situation be as in Setup [5.1. Then

$$
0_{H_{\mathfrak{m}}^{d}(R)}^{*}=\operatorname{Ann}_{H_{\mathfrak{m}}^{d}(R)} H^{0}\left(X, \omega_{X}\right)=\operatorname{Ker}\left(H_{\mathfrak{m}}^{d}(R) \stackrel{\delta}{\longrightarrow} H_{Z}^{d}\left(\mathcal{O}_{X}\right)\right),
$$

where $\delta$ is an edge map of the Leray spectral sequence as in 3.5. or dually,

$$
\tau\left(\omega_{R}\right)=H^{0}\left(X, \omega_{X}\right) \quad \text { in } \omega_{R} .
$$

Proof. By Corollary 3.5 , we only have to prove that $0_{H_{\mathrm{m}}^{d}(R)}^{*} \subseteq \operatorname{Ann}_{H_{\mathrm{m}}^{d}(R)} H^{0}\left(X, \omega_{X}\right)$. To see this we begin with the following assertion.

Claim. $H^{0}\left(X, \omega_{X}(\lceil n D\rceil)\right) \subseteq \tau\left(\omega_{R}\right)$ for all $n \gg 0$.

Proof of Claim. First note that if the localization $R_{c}$ at an element $c \in R^{\circ}$ is regular, then $\tau\left(\omega_{R}\right)_{c}=\omega_{R_{c}}$. Indeed, if $R_{c}$ is strongly $F$-regular, then some power $c^{n}$ of $c$ is a test element by 1.12 , so that $c^{n} \cdot 0_{H_{\mathfrak{m}}^{d}(R)}^{*}=c^{n} \cdot 0_{H_{\mathfrak{m}}^{d}(R)}^{* f g}=0$, whence $c^{n} \omega_{R} \subseteq$ $\operatorname{Ann}_{\omega_{R}}\left(0_{H_{\mathrm{m}}^{d}(R)}^{*}\right)=\tau\left(\omega_{R}\right)$. Thus, if $P \in \operatorname{Spec} R$ is not in $f(E)$, then $\tau\left(\omega_{R}\right)_{P}=$ $\omega_{R_{P}}=H^{0}\left(X, \omega_{X}\right)_{P}$. Let $\mathcal{R}=\bigoplus_{n \geq 0} J_{n}$ be the Rees algebra defined by the filtration $J_{n}=H^{0}\left(X, \mathcal{O}_{X}(n D)\right)$ on $R$. Since $D$ is $f$-ample, $\mathcal{K}=\bigoplus_{n>0} H^{0}\left(X, \omega_{X}(\lceil n D\rceil)\right)$ is a finitely generated $\mathcal{R}$-module. Say $\mathcal{K}$ is generated in degree $\leq n_{0}$. On the other hand, $J_{n_{1}} \cdot H^{0}\left(X, \omega_{X}\right) \subseteq \tau\left(\omega_{R}\right)$ for some $n_{1} \geq 0$, since $\operatorname{Supp}\left(H^{0}\left(X, \omega_{X}\right) / \tau\left(\omega_{R}\right)\right) \subseteq f(E)$. Therefore, if $n \geq n_{0}+n_{1}$, then $H^{0}\left(X, \omega_{X}(\lceil n D\rceil)\right) \subseteq \tau\left(\omega_{R}\right)$, as claimed.

Now, since $-D$ is effective, we have the natural map

$$
H_{\mathfrak{m}}^{d}(R) \stackrel{\delta}{\longrightarrow} H_{Z}^{d}\left(\mathcal{O}_{X}\right) \rightarrow H_{Z}^{d}\left(\mathcal{O}_{X}(-n D)\right)
$$

for each $n \geq 0$. As $H_{Z}^{d}\left(\mathcal{O}_{X}(-n D)\right)$ is the Matlis dual of $H^{0}\left(X, \omega_{X}(\lceil n D\rceil)\right)$, the kernel of this map turns to be

$$
\begin{aligned}
\operatorname{Filt}^{n}\left(H_{\mathfrak{m}}^{d}(R)\right) & :=\operatorname{Ann}_{H_{\mathfrak{m}}^{d}(R)} H^{0}\left(X, \omega_{X}(\lceil n D\rceil)\right) \\
& =\operatorname{Hom}_{R}\left(\frac{\omega_{R}}{H^{0}\left(X, \omega_{X}(\lceil n D\rceil)\right)}, E_{R}(R / \mathfrak{m})\right),
\end{aligned}
$$

as in the proof of Corollary 3.5. Thus we have the following commutative diagram with exact rows for each $q=p^{e}$ :

$$
\begin{aligned}
& \begin{array}{cccccc}
0 \rightarrow \operatorname{Filt}^{1}\left(H_{\mathfrak{m}}^{d}(R)\right) & \rightarrow & H_{\mathfrak{m}}^{d}(R) & \rightarrow & H_{Z}^{d}\left(\mathcal{O}_{X}\right) & \rightarrow 0 \\
\downarrow & & \downarrow F^{e} & & \downarrow F^{e} &
\end{array} \\
& 0 \rightarrow \operatorname{Filt}^{q}\left(H_{\mathfrak{m}}^{d}(R)\right) \rightarrow H_{\mathfrak{m}}^{d}(R) \rightarrow H_{Z}^{d}\left(\mathcal{O}_{X}(-q D)\right) \rightarrow 0
\end{aligned}
$$

Here the Frobenius map $F^{e}: H_{Z}^{d}\left(\mathcal{O}_{X}\right) \rightarrow H_{Z}^{d}\left(\mathcal{O}_{X}(-q D)\right)$ on the right-hand side is injective (5.1).

Now let $\xi \in H_{\mathfrak{m}}^{d}(R) \backslash \operatorname{Filt}^{1}\left(H_{\mathfrak{m}}^{d}(R)\right)$. Then $\xi^{q}:=F^{e}(\xi) \in H_{\mathfrak{m}}^{d}(R)$ does not lie in $\operatorname{Filt}^{q}\left(H_{\mathfrak{m}}^{d}(R)\right)=\operatorname{Ann}_{H_{\mathfrak{m}}^{d}(R)} H^{0}\left(X, \omega_{X}(\lceil q D\rceil)\right)$ for all $q=p^{e}$, by the above diagram. Hence the claim tells us that $\xi^{q} \notin \operatorname{Ann}_{H_{\mathrm{m}}^{d}(R)} \tau\left(\omega_{R}\right)$ for sufficiently large $q$. Since $0_{H_{\mathrm{m}}^{d}(R)}^{*}$ is contained in $\operatorname{Ann}_{H_{\mathrm{m}}^{d}(R)} \tau\left(\omega_{R}\right)$ and stable under the Frobenius action on $H_{\mathfrak{m}}^{d}(R)$, we have $\xi \notin 0_{H_{\mathfrak{m}}^{d}(R)}^{*}$. It follows that $0_{H_{\mathfrak{m}}^{d}(R)}^{*} \subseteq \operatorname{Filt}^{1}\left(H_{\mathfrak{m}}^{d}(R)\right)=$ 
$\operatorname{Ann}_{H_{\mathbf{m}}^{d}(R)} H^{0}\left(X, \omega_{X}\right)$, and that $\tau\left(\omega_{R}\right) \supseteq H^{0}\left(X, \omega_{X}\right)$ by Lemma 3.3 This completes the proof of the theorem.

5.3. Remark. (i) Theorem 5.2 implies that $0_{H_{\mathfrak{m}}^{d}(R)}^{*}=\operatorname{Ann}_{H_{\mathfrak{m}}^{d}(R)} A_{n_{\omega_{R}}}\left(0_{H_{\mathfrak{m}}^{d}(R)}^{*}\right)$ even though $(R, \mathfrak{m})$ is not complete. (This is trivial if $(R, \mathfrak{m})$ is complete; cf. Lemma 3.3.)

(ii) Since the surjectivity of the map $\left(F^{e}\right)^{\vee}$ is preserved under localization (4.6), Theorem 5.2 holds for all localizations of a finitely generated $\kappa$-algebra and its resolution reduced from characteristic zero to characteristic $p \gg 0$. This in particular means that the parameter test module commutes with localization under Setup 5.1, because so does the cohomology module $H^{0}\left(X, \omega_{X}\right)$. Namely, in characteristic $p \gg 0$ we have

$$
\tau\left(\omega_{R}\right)_{P}=\tau\left(\omega_{R_{P}}\right)
$$

for every $P \in \operatorname{Spec} R$. However, we expect that this is true in arbitrary characteristic, and our theorem does not answer the question. This problem is treated in LS2, [S1].

(iii) The argument in (ii) also tells us that the formula $\tau\left(\omega_{R}\right)=H^{0}\left(X, \omega_{X}\right)$ holds for a finitely generated $\kappa$-algebra in characteristic $p \gg 0$, where $\tau\left(\omega_{R}\right)$ of a non-local ring is defined as in 3.2 (ii).

Corollary 5.4 ([ $[\mathrm{Ha}$, Theorem 4.7]). In the situation of Setup [5.1, assume further that the non-(F-)rational locus of $\operatorname{Spec} R$ is isolated. Then

$$
H^{d-1}\left(X, \mathcal{O}_{X}\right) \cong 0_{H_{\mathfrak{m}}^{d}(R)}^{*}
$$

Proof. As we are working in characteristic $p \gg 0$, we have $H_{Z}^{d-1}\left(\mathcal{O}_{X}\right)=0$ (the dual Grauert-Riemenschneider vanishing), and

$$
H^{d-1}\left(X-Z, \mathcal{O}_{X-Z}\right) \cong H^{d-1}\left(\operatorname{Spec} R-\{\mathfrak{m}\}, \mathcal{O}_{\operatorname{Spec} R-\{\mathfrak{m}\}}\right) \cong H_{\mathfrak{m}}^{d}(R)
$$

since $\operatorname{Spec} R-\{\mathfrak{m}\}$ is rational. Hence we have an exact sequence

$$
0 \rightarrow H^{d-1}\left(X, \mathcal{O}_{X}\right) \rightarrow H_{\mathfrak{m}}^{d}(R) \stackrel{\delta}{\rightarrow} H_{Z}^{d}\left(\mathcal{O}_{X}\right) \rightarrow 0,
$$

which is the Matlis dual of $0 \rightarrow f_{*} \omega_{X} \rightarrow \omega_{R} \rightarrow \omega_{R} / f_{*} \omega_{X} \rightarrow 0$. Thus we see that

$$
H^{d-1}\left(X, \mathcal{O}_{X}\right) \cong \operatorname{Ker}(\delta)=0_{H_{\mathrm{m}}^{d}(R)}^{*} .
$$

5.5. Discussion. We consider another dual form of the formula $\tau\left(\omega_{R}\right)=H^{0}\left(X, \omega_{X}\right)$ under Setup 5.1 Let $D_{X}^{\bullet}$ and $D_{R}^{\bullet}$ denote the dualizing complexes of $X$ and $R$, respectively. Then $D_{X}^{\bullet}=\omega_{X}[d]$ since $X$ is Cohen-Macaulay. Note also that the Grauert-Riemenschneider vanishing in characteristic zero GR descends to our reduction modulo $p \gg 0$, i.e., $R^{i} f_{*} \omega_{X}=0$ for $i>0$. Therefore one has

$$
\mathbb{R} \operatorname{Hom}_{R}\left(\mathbb{R} f_{*} \mathcal{O}_{X}, D_{R}^{\bullet}\right)=\mathbb{R} f_{*}\left(\mathbb{R} \mathcal{H} \operatorname{com}_{\mathcal{O}_{X}}\left(\mathcal{O}_{X}, D_{X}^{\bullet}\right)\right)=\mathbb{R} f_{*} \omega_{X}[d]=f_{*} \omega_{X}[d]
$$

by the Grothendieck duality [Ht]. By dualizing this formula one has

$$
\mathbb{R} f_{*} \mathcal{O}_{X}=\mathbb{R} \operatorname{Hom}_{R}\left(f_{*} \omega_{X}, D_{R}^{\bullet}\right)[-d] .
$$

Theorem 5.2 allows us to replace $f_{*} \omega_{X}$ in the right-hand side by $\tau\left(\omega_{R}\right)$. 
Theorem 5.6. In the situation of Setup [5.1, assume further that $R$ is CohenMacaulay (with possibly non-isolated non-(F-)rational locus). Then

$$
H^{d-1}\left(X, \mathcal{O}_{X}\right) \cong \operatorname{Coker}\left(\bigoplus_{h t P=d-1} 0_{H_{P R_{P}}^{d-1}\left(R_{P}\right)}^{*} \rightarrow 0_{H_{\mathfrak{m}}^{d}(R)}^{*}\right),
$$

where the map $0_{H_{P R_{P}}^{d-1}\left(R_{P}\right)}^{*} \rightarrow 0_{H_{\mathfrak{m}}^{d}(R)}^{*}$ is induced by the map $E_{R}(R / P) \rightarrow E_{R}(R / \mathfrak{m})$ in the minimal injective resolution of the $R$-module $\omega_{R}$.

Proof. Since $R$ is Cohen-Macaulay, $D_{R}^{\bullet}=\omega_{R}[d]$, so that by 5.5 one has

$$
\mathbb{R} f_{*} \mathcal{O}_{X}=\mathbb{R H o m} R\left(\tau\left(\omega_{R}\right), \omega_{R}\right) .
$$

Since $\omega_{R}$ is also a Cohen-Macaulay $R$-module, it follows that

$$
\begin{aligned}
R^{i} f_{*} \mathcal{O}_{X} & =\operatorname{Ext}_{R}^{i}\left(\tau\left(\omega_{R}\right), \omega_{R}\right) \\
& =\operatorname{Ext}_{R}^{i+1}\left(\omega_{R} / \tau\left(\omega_{R}\right), \omega_{R}\right)=H^{i+1}\left(\operatorname{Hom}_{R}\left(\omega_{R} / \tau\left(\omega_{R}\right), I^{\bullet}\right)\right)
\end{aligned}
$$

for $i>0$, where $I^{\bullet} \cong D_{R}^{\bullet}[-d]$ is the minimal injective resolution of $\omega_{R}$. The theorem is proved, because the $i$ th spot of the complex $\operatorname{Hom}_{R}\left(\omega_{R} / \tau\left(\omega_{R}\right), I^{\bullet}\right)$ is

$$
\operatorname{Hom}_{R}\left(\omega_{R} / \tau\left(\omega_{R}\right), \bigoplus_{\mathrm{ht} P=i} E_{R_{P}}\left(R_{P} / P R_{P}\right)\right)=\bigoplus_{\mathrm{ht} P=i} 0_{H_{P R_{P}}^{i}\left(R_{P}\right)}^{*}
$$

by Remark 5.3 .

5.7. Discussion. We now generalize Theorem5.2 in terms of cyclic covering. To do this we again start from characteristic zero, and then move to reduction modulo $p$ as in 4.6 .

Let $R$ be a normal finitely generated algebra over a field of characteristic zero, and let $I$ be a divisorial ideal such that $I^{(n)} \cong R$ for some $n \in \mathbb{N}$. Let $D$ be the $\mathbb{Q}$-Cartier Weil divisor on $\operatorname{Spec} R$ corresponding to $I$ (i.e., $\left.I=H^{0}(\operatorname{Spec} R, \mathcal{O}(D))\right)$, and let $f: X \rightarrow \operatorname{Spec} R$ be a resolution of singularities whose exceptional set $E \subset X$ is a simple normal crossing divisor. Since $n D$ is a Cartier divisor, one can define the pull-back of $D$ by $f$ to be the $\mathbb{Q}$-divisor $f^{*} D:=\frac{1}{n} f^{*}(n D)$. Note that the fractional part $f^{*} D-\left\lfloor f^{*} D\right\rfloor$ of $f^{*} D$ is supported in $E$.

For a fixed isomorphism $I^{(n)} \cong R$, we consider the corresponding cyclic coverings $\pi: \operatorname{Spec} S \rightarrow \operatorname{Spec} R$ and $\pi^{\prime}: Y \rightarrow X$, where $S=\bigoplus_{i=0}^{n-1} I^{(i)}$ and $Y=$ $\operatorname{Spec}_{X}\left(\bigoplus_{i=0}^{n-1} \mathcal{O}_{X}\left(\left\lfloor i f^{*} D\right\rfloor\right)\right)$. Let $h: \tilde{Y} \rightarrow Y$ be a resolution of singularities. Since $X$ is smooth and the ramification divisor $\operatorname{Supp}\left(f^{*} D-\left\lfloor f^{*} D\right\rfloor\right)$ of $\pi^{\prime}: Y \rightarrow X$ is simple normal crossing, $Y$ has rational singularities. (Even more, $Y$ has toric singularities; cf. [EV, Lemma 3.24], [TW].) By the adjunction formula we also have

$$
h_{*} \omega_{\tilde{Y}}=\omega_{Y}=\mathcal{H} m_{\mathcal{O}_{X}}\left(\mathcal{O}_{Y}, \omega_{X}\right)=\bigoplus_{i=0}^{n-1} \omega_{X}\left(\left\lceil i f^{*} D\right\rceil\right) .
$$

On the other hand, the natural map $H^{0}\left(X, \mathcal{O}_{X}\left(\left\lfloor i f^{*} D\right\rfloor\right)\right) \hookrightarrow I^{(i)}$ is an isomorphism for each $i$. Indeed, if $\varphi \in I^{(i)}$, then $\varphi^{n} \in I^{(i n)}=\left(I^{(n)}\right)^{i}=H^{0}\left(X, \mathcal{O}_{X}\left(i f^{*}(n D)\right)\right)$, so that $\operatorname{div}_{X}\left(\varphi^{n}\right)+i f^{*}(n D) \geq 0$. Dividing out by $n$ gives $\operatorname{div}_{X}(\varphi)+\left\lfloor i f^{*} D\right\rfloor \geq 0$, since $\operatorname{div}_{X}(\varphi)$ has integer coefficients, so $\varphi \in H^{0}\left(X, \mathcal{O}_{X}\left(\left\lfloor i f^{*} D\right\rfloor\right)\right)$. Thus $S=H^{0}\left(Y, \mathcal{O}_{Y}\right)$, and this gives rise to a proper birational morphism $g: Y \rightarrow \operatorname{Spec} S$. The natural inclusion $H^{0}\left(Y, \omega_{Y}\right) \rightarrow \omega_{S}$ induced by $g$ preserves the $\mathbb{Z}_{n}$-grading of $H^{0}\left(\tilde{Y}, \omega_{\tilde{Y}}\right)=$ $H^{0}\left(Y, \omega_{Y}\right)=\bigoplus_{i=0}^{n-1} H^{0}\left(X, \omega_{X}\left(\left\lceil i f^{*} D\right\rceil\right)\right)$ and $\omega_{S}=\bigoplus_{i=0}^{n-1} \operatorname{Hom}_{R}\left(I^{(-i)}, \omega_{R}\right)$. 
Now we consider the following commutative diagram in characteristic zero:

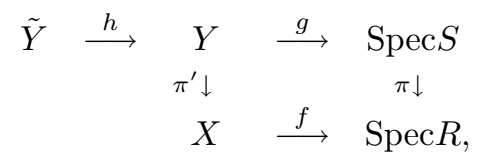

and reduce all the data to characteristic $p \gg 0$. We switch the notation to denote sufficiently general reduction modulo $p$ (or localization thereof as in 5.1), and assume in the sequel that the above diagram is defined over a perfect field $\kappa$ of characteristic $p>0$ which does not divide $n$.

Theorem 5.8. In the situation of [5.7, let $(R, \mathfrak{m})$ be a normal local ring of dimension $d$ which is reduced from characteristic zero to characteristic $p \gg 0$, together with the divisorial ideal $I=H^{0}(\operatorname{Spec} R, \mathcal{O}(D))$, the resolution of singularities $f: X \rightarrow \operatorname{Spec} R$, etc. Let $Z$ denote the closed fiber of $f$. Then

$$
0_{H_{\mathfrak{m}}^{d}(I)}^{*}=\operatorname{Ker}\left(H_{\mathfrak{m}}^{d}(I) \stackrel{\delta}{\longrightarrow} H_{Z}^{d}\left(\mathcal{O}_{X}\left(\left\lfloor f^{*} D\right\rfloor\right)\right)\right),
$$

where $\delta$ denotes an edge map of the Leray spectral sequence $H_{\mathfrak{m}}^{i}\left(H^{j}\left(X, \mathcal{O}_{X}\left(\left\lfloor f^{*} D\right\rfloor\right)\right)\right)$ $\Longrightarrow H_{Z}^{i+j}\left(\mathcal{O}_{X}\left(\left\lfloor f^{*} D\right\rfloor\right)\right)$.

Proof. Since $(R, \mathfrak{m})$ is local, $S$ is semilocal. Let $\mathfrak{n}_{1}, \ldots, \mathfrak{n}_{s}$ be the maximal ideals of $S$, and $Z_{i}\left(\right.$ resp. $\left.\tilde{Z}_{i}\right)$ the fiber of $g: Y \rightarrow \operatorname{Spec} S$ (resp. $g \circ h: \tilde{Y} \rightarrow \operatorname{Spec} S$ ) over $\mathfrak{n}_{i}$. Since $R^{j} h_{*} \mathcal{O}_{\tilde{Y}}=0$ for $j>0$, we have $0_{H_{\mathfrak{n}_{i}}^{d}(S)}^{*}=\operatorname{Ker}\left(H_{\mathfrak{n}_{i}}^{d}(S) \rightarrow H_{\tilde{Z}_{i}}^{d}\left(\mathcal{O}_{\tilde{Y}}\right)=\right.$ $\left.H_{Z_{i}}^{d}\left(\mathcal{O}_{Y}\right)\right)$ by Theorem 5.2 So, denoting $\mathfrak{n}=\mathfrak{n}_{1} \cap \cdots \cap \mathfrak{n}_{s}$ and $Z^{\prime}=Z_{1} \cup \cdots \cup Z_{s}$, we have

$$
0_{H_{\mathfrak{n}}^{d}(S)}^{*}=\operatorname{Ker}\left(H_{\mathfrak{n}}^{d}(S) \stackrel{\delta^{\prime}}{\longrightarrow} H_{Z^{\prime}}^{d}\left(\mathcal{O}_{Y}\right)\right)
$$

where $\delta^{\prime}$ is an edge map of the spectral sequence $H_{\mathfrak{n}}^{i}\left(H^{j}\left(Y, \mathcal{O}_{Y}\right)\right) \Longrightarrow H_{Z^{\prime}}^{i+j}\left(\mathcal{O}_{Y}\right)$ induced from $\Gamma_{Z^{\prime}}=\Gamma_{\mathfrak{n}} \circ g_{*}$. This map $\delta^{\prime}$, being the dual map of the $\mathbb{Z}_{n}$-graded map $H^{0}\left(Y, \omega_{Y}\right) \rightarrow \omega_{S}$ with respect to the $\mathbb{Z}_{n}$-graded module $E_{S}(S / \mathfrak{n})$, is also $\mathbb{Z}_{n}$-graded, and its degree 1 part is

$$
\delta: H_{\mathfrak{m}}^{d}(I)=H_{\mathfrak{m}}^{d}\left(H^{0}\left(X, \mathcal{O}_{X}\left(\left\lfloor f^{*} D\right\rfloor\right)\right)\right) \rightarrow H_{Z}^{d}\left(\mathcal{O}_{X}\left(\left\lfloor f^{*} D\right\rfloor\right)\right),
$$

since $\Gamma_{\mathfrak{n}}=\Gamma_{\mathfrak{m}} \circ \pi_{*}$ and $\Gamma_{Z^{\prime}}=\Gamma_{Z} \circ \pi_{*}^{\prime}$. On the other hand, the degree 1 part of $0_{H_{\mathfrak{n}}^{d}(S)}^{*}$ in the $\mathbb{Z}_{n}$-grading is $0_{H_{\mathfrak{m}}^{d}(I)}^{*}$ by Proposition 3.7 This completes the proof of the theorem.

The most important application of Theorem 5.8 is the case $I \cong \omega_{R}$, as follows.

Theorem 5.9. Let $R$ be a normal $\mathbb{Q}$-Gorenstein finitely generated $\kappa$-algebra (or its local ring) which is reduced from characteristic zero to characteristic $p \gg 0$, together with a resolution of singularities $f: X \rightarrow \operatorname{Spec} R$ whose exceptional set $E \subset X$ is a simple normal crossing divisor, as in 5.7. Let $\Delta=K_{X}-f^{*} K_{R}$ be the discrepancy of $f$. Then the test ideal $\tau(R)$ of $R$ is described as

$$
\tau(R)=H^{0}\left(X, \mathcal{O}_{X}(\lceil\Delta\rceil)\right),
$$

where the right-hand side is viewed as an ideal of $R$ in terms of the natural inclusion map $H^{0}\left(X, \mathcal{O}_{X}(\lceil\Delta\rceil)\right) \rightarrow H^{0}\left(X-E, \mathcal{O}_{X}(\lceil\Delta\rceil)\right)=R$. 
Proof. We first consider the case when $(R, \mathfrak{m})$ is local, and apply Theorem 5.8 to the case $D=K_{R}$. Then we have $f^{*} D=K_{X}-\Delta$, and $H_{Z}^{d}\left(\mathcal{O}_{X}\left(\left\lfloor K_{X}-\Delta\right\rfloor\right)\right)=$ $H_{Z}^{d}\left(\omega_{X}(-\lceil\Delta\rceil)\right)$ is the Matlis dual of $H^{0}\left(X, \mathcal{O}_{X}(\lceil\Delta\rceil)\right)$. Hence the tight closure of zero in $E_{R}(R / \mathfrak{m}) \cong H_{\mathfrak{m}}^{d}\left(\omega_{R}\right)$ is described as

$0_{H_{\mathfrak{m}}^{d}\left(\omega_{R}\right)}^{*}=\operatorname{Ker}\left(H_{\mathfrak{m}}^{d}\left(\omega_{R}\right) \rightarrow H_{Z}^{d}\left(\mathcal{O}_{X}\left(\left\lfloor K_{X}-\Delta\right\rfloor\right)\right)\right)=\operatorname{Ann}_{H_{\mathfrak{m}}^{d}\left(\omega_{R}\right)} H^{0}\left(X, \mathcal{O}_{X}(\lceil\Delta\rceil)\right)$.

Therefore we have

$$
\tau(R)=\operatorname{Ann}_{R}\left(0_{H_{\mathrm{m}}^{d}\left(\omega_{R}\right)}^{*}\right)=H^{0}\left(X, \mathcal{O}_{X}(\lceil\Delta\rceil)\right),
$$

by Theorem 1.8. This equality holds even when $R$ is not local, because it is true for all localizations at maximal ideals of $R$ (cf. 5.3 (iii)).

5.10. Remark. (i) We have to assume in Theorem 5.9 that $R$ is $\mathbb{Q}$-Gorenstein, i.e., $\omega_{R}^{(n)}$ is locally free for some $n \in \mathbb{N}$. But in fact, $\omega_{R}^{(n)}$ need not be globally free.

(ii) When $\omega_{R} \cong R$, the assertions in Theorems 5.2 and 5.9 exactly coincide with each other via the identification $\omega_{R} \cong R$.

(iii) The right-hand side of Theorem 5.9 is the multiplier ideal as in 4.2. From this theorem we easily rediscover the equivalence of log terminal singularity and $F$-regular type under the $\mathbb{Q}$-Gorenstein property.

Corollary 5.11. Let $R$ be a normal $\mathbb{Q}$-Gorenstein finitely generated $\kappa$-algebra (or its local ring) which is reduced from characteristic zero to characteristic $p \gg 0$, as in 5.9. Then the test ideal $\tau(R)$ of $R$ is integrally closed, and so $\tau(R)$ is tightly closed.

Corollary 5.12. Let the situation be as in Theorem 5.9 and assume $R=(R, \mathfrak{m})$ is a local ring of dimension $d \geq 2$. Then:

(i) If $\operatorname{Spec} R \backslash\{\mathfrak{m}\}$ is F-regular, then $H^{d-1}\left(X, \omega_{X}(\lfloor-\Delta\rfloor)\right) \cong 0_{E_{R}(R / \mathfrak{m})}^{*}$.

(ii) If $R$ is Cohen-Macaulay, then

$$
H^{d-1}\left(X, \omega_{X}(\lfloor-\Delta\rfloor)\right) \cong \operatorname{Coker}\left(\bigoplus_{\mathrm{ht} P=d-1} 0_{E_{R}(R / P)}^{*} \rightarrow 0_{E_{R}(R / \mathfrak{m})}^{*}\right),
$$

where the map $0_{E_{R}(R / P)}^{*} \rightarrow 0_{E_{R}(R / \mathfrak{m})}^{*}$ is induced by the map $E_{R}(R / P) \rightarrow E_{R}(R / \mathfrak{m})$ in the minimal injective resolution of the $R$-module $\omega_{R}$.

Proof. Since we are working in characteristic $p \gg 0$, we have $R^{i} f_{*} \mathcal{O}_{X}(\lceil\Delta\rceil)=$ $R^{i} f_{*} \omega_{X}\left(\left\lceil-f^{*} K_{R}\right\rceil\right)=0$ for $i>0$ by the Kawamata-Viehweg vanishing theorem in characteristic zero [KMM]. Then the similar argument as in the proof of Corollary 5.4 and Theorem 5.6 works.

5.13. Remark. In case (ii) of Corollary 5.12, we have shown that

$$
\mathbb{R} f_{*} \omega_{X}(\lfloor-\Delta\rfloor)=\mathbb{R A B S}\left(\omega_{R}\right),
$$

where ABS is the left exact functor defined in [HH1, 8.25].

\section{The GRADED CASE}

In this section we restrict ourselves to the case where $R$ is an $\mathbb{N}$-graded ring, and reformulate the results in the previous section in terms of the graded structure.

We first interpret Corollary 5.4 in the graded case. 
6.1. Discussion. Let $R=\bigoplus_{n \geq 0} R_{n}$ be a normal graded $\operatorname{ring}$ with $\operatorname{dim} R=d \geq 2$ over a field $k=R_{0}$ of characteristic zero, and let $\mathfrak{m}=R_{+}$be the graded maximal ideal. Let $\varphi: Y=\operatorname{Proj}\left(\bigoplus_{n \geq 0} R_{\geq n}\right) \rightarrow$ Spec $R$ be the blowing-up with respect to the filtration of ideals $R_{\geq n} \stackrel{=}{=} \bigoplus_{m \geq n} R_{m} \subseteq R(n=0,1,2, \ldots)$. (We call $Y$ the graded blowing-up of Spec $R$.) Also let $\psi: \tilde{Y} \rightarrow Y$ be a resolution of singularities of $Y$. If $\operatorname{Spec} R \backslash\{\mathfrak{m}\}$ has only rational singularities, then $Y$ also has only rational singularities by [W1, Lemma 2.4]. So, by the Leray spectral sequence, we have

$$
H^{d-1}\left(\tilde{Y}, \mathcal{O}_{\tilde{Y}}\right)=H^{d-1}\left(Y, \mathcal{O}_{Y}\right)=H_{\mathfrak{m}}^{d}(R)_{\geq 0},
$$

where $H_{\mathfrak{m}}^{d}(R)_{\geq 0}=\bigoplus_{n \geq 0} H_{\mathfrak{m}}^{d}(R)_{n}$ is the nonnegative graded part of the graded $R$ module $H_{\mathfrak{m}}^{d}(R)$. Thus, Corollary 5.4 says that, after moving to reduction modulo $p \gg 0$,

$$
0_{H_{\mathfrak{m}}^{d}(R)}^{*}=H_{\mathfrak{m}}^{d}(R)_{\geq 0} .
$$

This is reformulated as follows.

Proposition 6.2 ([FW], [HS] $)$. Let $R=\bigoplus_{n \geq 0} R_{n}$ be a graded ring of $\operatorname{dim} R=$ $d \geq 2$ over a perfect field $k=R_{0}$ of characteristic $p>0$, and assume that $\operatorname{Spec} R$ is $F$-rational off the vertex $\mathfrak{m}=R_{+}$. Then the following conditions are equivalent:

(i) $R$ is F-injective in negative degree, i.e., the Frobenius map acts injectively on the negative graded part $H_{\mathfrak{m}}^{d}(R)_{<0}$ of $H_{\mathfrak{m}}^{d}(R)$.

(ii) $0_{H_{\mathfrak{m}}^{d}(R)}^{*}=H_{\mathfrak{m}}^{d}(R)_{\geq 0}$.

(iii) For every homogeneous system of parameters $x_{1}, \ldots, x_{d}$ of $R$, one has

$$
\left(x_{1}, \ldots, x_{d}\right)^{*}=\left(x_{1}, \ldots, x_{d}\right)^{\lim }+R_{\geq \delta},
$$

where $\left(x_{1}, \ldots, x_{d}\right)^{\lim }$ is the "limit closure" of $\left(x_{1}, \ldots, x_{d}\right)$ as defined in [HS], and $\delta=\sum_{i=1}^{d} \operatorname{deg} x_{i}$.

(iv) $\tau\left(\omega_{R}\right)=\operatorname{Ann}_{\omega_{R}}\left(0_{H_{\mathrm{m}}(R)}^{*}\right)$ coincides with the positive graded part $\left[\omega_{R}\right]_{>0}$ of the canonical module $\omega_{R}$.

6.3. Remark. Huneke and Smith $\mathrm{HS}$ conjectured that condition (iii) in Proposition 6.2 holds true if $R$ is a reduction modulo $p \gg 0$ of a graded ring of characteristic zero (or, $R$ has characteristic zero). They called this the "Vanishing Conjecture," and showed that it is related to the Kodaira vanishing theorem. As is mentioned in 6.1, the results in Section 5 gives an affirmative answer to the Vanishing Conjecture. Here is another way to prove it, which we outline very briefly.

By $\mathbb{D}$, any normal graded ring $R$ is represented by $X=\operatorname{Proj} R$ and an ample $\mathbb{Q}$ Cartier divisor $D$ on $X$ as $R \cong R(X, D)=\bigoplus_{n \geq 0} H^{0}\left(X, \mathcal{O}_{X}(n D)\right) T^{n}$. In this manner the top local cohomology is written as $H_{\mathfrak{m}}^{d}(R)=\bigoplus_{n \in \mathbb{Z}} H^{d-1}\left(X, \mathcal{O}_{X}(n D)\right) T^{n}$. Therefore, to check condition (i) of Proposition 6.2 , we may prove that the induced Frobenius map

$$
F: H^{d-1}\left(X, \mathcal{O}_{X}(n D)\right) \rightarrow H^{d-1}\left(X, \mathcal{O}_{X}(p n D)\right)
$$

is injective for all $n<0$. If $(X, D)$ is reduced from characteristic zero to characteristic $p \gg 0$, this injectivity follows from the vanishing theorems of Akizuki-Nakano and Serre by [Ha, Proposition 3.5] as in 4.6.

Next we reinterpret the test ideal in the graded case: 
Proposition 6.4. Let $R=\bigoplus_{n \geq 0} R_{n}$ be a normal graded ring with $\operatorname{dim} R=d \geq 2$ over a perfect field $k=R_{0}$ of characteristic $p>0$. Assume that $\omega_{R}^{(r)} \cong R(b)$ for some integers $r>0$ and $b \in \mathbb{Z}$. Also assume that $\operatorname{Spec} R$ is (strongly) F-regular off the vertex $\mathfrak{m}=R_{+}$. Then the following are equivalent:

(i) The Frobenius map $F: H_{\mathfrak{m}}^{d}\left(\omega_{R}^{(i)}\right)_{n} \rightarrow H_{\mathfrak{m}}^{d}\left(\omega_{R}^{(p i)}\right)_{p n}$ induced on the degree $n$ part of $H_{\mathfrak{m}}^{d}\left(\omega_{R}^{(i)}\right)$ is injective for all $i=0, \ldots, r-1$ and $n<-b i / r$.

(ii) $0_{H_{\mathfrak{m}}^{d}\left(\omega_{R}^{(i)}\right)}^{*}=H_{\mathfrak{m}}^{d}\left(\omega_{R}^{(i)}\right)_{\geq-b i / r}$ for all $i=0, \ldots, r-1$.

In particular, if the above equivalent conditions hold, then

$$
\tau(R)=R_{>b / r}
$$

Proof. First note that ${ }^{e} R \otimes_{R} H_{\mathfrak{m}}^{d}\left(\omega_{R}\right) \cong H_{\mathfrak{m}}^{d}\left(\omega_{R}^{(q)}\right)$ for each $q=p^{e}$ by [W2]. Then the implication (ii) $\Rightarrow$ (i) is obvious. For the converse implication, we fix an $i$ with $0 \leq i \leq r-1$, and first show that $0_{H_{\mathfrak{m}}^{d}\left(\omega_{R}^{(i)}\right)}^{*} \supseteq H_{\mathfrak{m}}^{d}\left(\omega_{R}^{(i)}\right)_{\geq-b i / r}$. Let $\xi \in H_{\mathfrak{m}}^{d}\left(\omega_{R}^{(i)}\right)_{n}$ for $n \geq-b i / r$. For a power $q=p^{e}$, write $q i=r s+j$ for integers $s$ and $j$ with $0 \leq j \leq r-1$. Then $\xi^{q} \in H_{\mathfrak{m}}^{d}\left(\omega_{R}^{(q i)}\right)_{q n} \cong H_{\mathfrak{m}}^{d}\left(\omega_{R}^{(j)}\right)_{b s+q n}$, since $\omega_{R}^{(q i)} \cong \omega_{R}^{(j)}(b s)$ as graded $R$-modules. Since $b s+q n>-b$, it follows that $\bigcap_{q=p^{e}} \operatorname{Ann}_{R}\left(\xi^{q}\right)$ contains $\bigcap_{j=0}^{r-1} \operatorname{Ann}_{R}\left(H_{\mathfrak{m}}^{d}\left(\omega_{R}^{(j)}\right)_{>-b}\right)$, which intersects $R^{\circ}$. This implies that $\xi \in 0_{H_{\mathfrak{m}}^{d}\left(\omega_{R}^{(i)}\right)}^{*}$.

Now suppose condition (i) holds and let $0 \neq \xi \in H_{\mathfrak{m}}^{d}\left(\omega_{R}^{(i)}\right)_{n}$ for $n<-b i / r$. If we write $q i=r s+j$ for $0 \leq j \leq r-1$ as above, then by the assumption we have that $0 \neq \xi^{q} \in H_{\mathfrak{m}}^{d}\left(\omega_{R}^{(q i)}\right)_{q n} \cong H_{\mathfrak{m}}^{d}\left(\omega_{R}^{(j)}\right)_{b s+q n}$, where $b s+q n \leq-q / r \rightarrow-\infty$ as $q \rightarrow \infty$. For each $q=p^{e}$, choose $c_{e} \in R^{\circ}$ such that $c_{e} \xi^{q}$ is a nonzero element of the socle of $H_{\mathfrak{m}}^{d}\left(\omega_{R}^{(q i)}\right) \cong H_{\mathfrak{m}}^{d}\left(\omega_{R}^{(j)}\right)(b s)$. Since the socles of $H_{\mathfrak{m}}^{d}(R)=H_{\mathfrak{m}}^{d}\left(\omega_{R}^{(0)}\right), \ldots, H_{\mathfrak{m}}^{d}\left(\omega_{R}^{(r-1)}\right)$ are finitely generated, they sit in degree $\geq n_{0}$ for some integer $n_{0}$. Then $\operatorname{deg} c_{e} \geq$ $n_{0}+q / r \rightarrow \infty$ as $q=p^{e} \rightarrow \infty$. This impies that $c_{e}$ is a test element of $R$ if $e$ is sufficiently large, since the test ideal $\tau(R)$ is $\mathfrak{m}$-primary by Theorem 1.12 (i). Hence it follows from $c_{e} \xi^{q} \neq 0$ that $\xi \notin 0_{H_{\mathfrak{m}}^{d}\left(\omega_{R}^{(i)}\right)}^{* f g}=0_{H_{\mathfrak{m}}^{d}\left(\omega_{R}^{(i)}\right)}^{*}$. Thus we have $0_{H_{\mathfrak{m}}^{d}\left(\omega_{R}^{(i)}\right)}^{*} \subseteq H_{\mathfrak{m}}^{d}\left(\omega_{R}^{(i)}\right)_{\geq-b i / r}$, proving (i) $\Rightarrow$ (ii).

The last assertion $\tau(R)=R_{>b / r}$ is just a dual form of $0_{H_{\mathfrak{m}}^{d}\left(\omega_{R}\right)}^{*}=H_{\mathfrak{m}}^{d}\left(\omega_{R}\right)_{\geq-b / r}$, since $H_{\mathfrak{m}}^{d}\left(\omega_{R}\right) \cong E_{R}(R / \mathfrak{m})$.

6.5. Remark. (i) The injectivity of the Frobenius maps in (i) of the above theorem can be proved in characteristic $p \gg 0$ as in 6.3 . Hence in characteristic $p \gg 0$, the test ideal of a graded ring $R$ as in Theorem 6.4 is of the form $\tau(R)=R_{>b / r}$. This implies that the multiplier ideal of such a ring is also described in this form.

(ii) In the situation of Proposition 6.4, the discrepancy of the unique exceptional divisor of the graded blowing-up $\varphi: Y \rightarrow \operatorname{Spec} R$ is $-1-b / r$. When $R$ is quasiGorenstein (i.e., $r=1), b / r$ is nothing but the $a$-invariant $a=a(R)$ of $R$, and the description of the test ideal $\tau(R)=R_{\geq a+1}$ is given in [HS].

(iii) The proof of Proposition 6.4 we presented above is a modification of the original proof of Proposition 6.2 given by Fedder and Watanabe [FW]. Similarly, we can prove Theorem 5.8 directly by modifying the proof of Theorem 5.2 . The advantage of this alternative proof is that we do not have to use cyclic covering (at least apparently). But it is somewhat laborious, and we do not present it here. On the other hand, when the index $r$ is not divisible by $p, 6.4$ is directly reduced to 6.2 
by the canonical cover technique. This "second proof" of Proposition 6.4, which we will see below, seems worth recording.

In the situation of 6.4 assume that $r$ is not divisible by $p$, and let $S=\bigoplus_{i=0}^{r-1} \omega_{R}^{(i)}$ be a canonical covering of $R$. Since $R$ is strongly $F$-regular off the vertex, so is $S$, too ([W2], see also [NW] for the case $p \mid r$ ). Taking into account the multiplicative structure of $S$ and the graded isomorphism $\omega_{R}^{(r)} \cong R(b)$, we deduce that $S$ also has a structure of an $\mathbb{N}$-graded ring given by $S_{n} \cap \omega_{R}^{(i)}=\left[\omega_{R}^{(i)}\right]_{\geq-b i / r}$ for $n \geq 0$ and $i=0, \ldots, r-1$, where $\omega_{R}^{(i)}$ is considered to be the $i$ th $\mathbb{Z}_{r}$-graded piece of $S$. Let $\mathfrak{n}=S_{+}=\bigoplus_{n>0} S_{n}$ be the $\mathbb{N}$-graded maximal ideal of $S$. Then we have

$$
H_{\mathfrak{n}}^{d}(S)_{\geq 0}=H_{\mathfrak{m}}^{d}(S)_{\geq 0}=\bigoplus_{i=0}^{r-1} H_{\mathfrak{m}}^{d}\left(\omega_{R}^{(i)}\right)_{\geq-b i / r}
$$

Hence conditions (i) and (ii) of Proposition 6.4 respectively correspond to those in Proposition 6.2 for $S$. (Here we need the assumption that $r$ is not divisible by $p$, to apply Proposition 3.7)

6.6. Example. Let us consider the case when $R=R(X, D)$ is a 2-dimensional normal graded ring with $\omega_{R}^{(r)} \cong R(b)$. Then $X=\operatorname{Proj} R$ is a smooth curve, and $r\left(K_{X}+D^{\prime}\right)-b D$ is a principal divisor, where $D^{\prime}$ is the "fractional part" of the $\mathbb{Q}$-divisor $D$ as defined in [W1]. In particular, $b / r=\operatorname{deg}\left(K_{X}+D^{\prime}\right) / \operatorname{deg} D$. Also, the Frobenius map of Proposition 6.4(i) is identified with the Frobenius

$$
F: H^{1}\left(X, \mathcal{O}_{X}\left(i\left(K_{X}+D^{\prime}\right)+n D\right)\right) \rightarrow H^{1}\left(X, \mathcal{O}_{X}\left(p\left(i\left(K_{X}+D^{\prime}\right)+n D\right)\right)\right) .
$$

If $n<-b i / r$, then $\operatorname{deg}\left(i\left(K_{X}+D^{\prime}\right)+n D\right)<0$, so that by [HW, Theorem 2.3], the above Frobenius map is injective if chark $=p$ is greater than a certain bound given explicitly by $(X, D)$.

(i) Let $X$ be a smooth curve of genus $g \geq 2$ and characteristic $p>0$, and let $D=r K_{X}$ for an integer $r \geq 2$. Then the geometric genus of $R=R(X, D)$ is $p_{g}(R)=g$, which can take arbitrary integer values $\geq 2$ as $X$ varies. On the other hand, we always have $\tau(R)=R_{>1 / r}=\mathfrak{m}$.

(ii) Let $X=\mathbb{P}_{k}^{1}$, a smooth rational curve with $\operatorname{char} k=p$, and let

$$
D=(1 / 3 n)\left(P_{1}+P_{2}+P_{3}\right)
$$

for a positive integer $n$ and different points $P_{1}, P_{2}, P_{3} \in X$. Then $R=R(X, D)$ has a rational singularity, i.e., $p_{g}(R)=0$. Also assume that $p>3(n-1)$. Then $R=R(X, D)$ is $F$-rational, i.e., $\tau_{\text {par }}(R)=R$ and $\tau\left(\omega_{R}\right)=\omega_{R}$. On the other hand, the test ideal is $\tau(R)=R_{\geq n}$.

\section{Appendix: Proof of Theorem 1.9}

In this appendix we prove Theorem 1.9 according to the method of MacCrimmon $\underline{\mathrm{MC}}$ and Williams $\mathrm{Wi}$. First, we recall

Theorem 1.9. Let $(R, \mathfrak{m})$ be a d-dimensional excellent normal local ring of characteristic $p>0$ and let $J \subseteq R$ be a divisorial ideal such that the divisor class $\operatorname{cl}(J) \in \mathrm{Cl}(R)$ has a finite order. Then $0_{H_{\mathfrak{m}}^{d}(J)}^{*}=0_{H_{\mathfrak{m}}^{d}(J)}^{* g}$.

To prove this theorem, we borrow some notation and preliminary results (with slight modification) from $\underline{\mathrm{Mc}}$ and $[\mathrm{Wi}$. 
A.1. Notation. Let $R$ be a Noetherian ring and $M$ an $R$-module. For a sequence of elements $\mathbf{x}=x_{1}, \ldots, x_{d}$ of $R$ and a positive integer $t$, we write $\mathbf{x}^{t}=x_{1}^{t}, \ldots, x_{d}^{t}$, and denote the kernel of the map $H^{d}(\mathbf{x} ; M) \rightarrow H^{d}\left(\mathbf{x}^{t} ; M\right)$ induced for the Koszul cohomologies by

$$
\mathcal{K}(\mathbf{x}, t, M):=\operatorname{Ker}\left(\frac{M}{(\mathbf{x}) M} \stackrel{\left(x_{1} \cdots x_{d}\right)^{t-1}}{\longrightarrow} \frac{M}{\left(\mathbf{x}^{t}\right) M}\right) .
$$

We also write

$$
\mathcal{K}(\mathbf{x}, \infty, M)=\bigcup_{t \in \mathbb{N}} \mathcal{K}(\mathbf{x}, t, M) .
$$

Theorem A.2 (Williams Wi]). Let $(R, \mathfrak{m})$ be a d-dimensional normal local ring of characteristic $p>0$, and let $J \subseteq R$ be a divisorial ideal. Let $\mathbf{x}=x_{1}, \ldots, x_{d}$ be a system of parameters of $R$. Suppose that there exist an element $c \in R^{\circ}$ and an integer $t_{0} \geq 2$ such that

$$
c \mathcal{K}\left(\mathbf{x}^{q s}, \infty, J^{[q]}\right) \subseteq \mathcal{K}\left(\mathbf{x}^{q s}, t_{0}, J^{[q]}\right)
$$

for all $s \geq 1$ and $q=p^{e} \gg 0$. Then $0_{H_{\mathrm{m}}^{d}(J)}^{*}=0_{H_{\mathrm{m}}^{d}(J)}^{* f g}$.

Proof. Let $\zeta \in 0_{H_{\mathfrak{m}}^{d}(J)}^{*}$, i.e., there exists $d \in R^{\circ}$ such that $d \zeta^{q}=0 \in{ }^{e} R \otimes_{R} H_{\mathfrak{m}}^{d}(J)$ for $q=p^{e} \gg 0$. Since $H_{\mathfrak{m}}^{d}(J) \cong \lim J /\left(\mathbf{x}^{t}\right) J, \zeta \in H_{\mathfrak{m}}^{d}(J)$ is represented by $z \bmod$ $\left(\mathbf{x}^{s}\right) \in J /\left(\mathbf{x}^{s}\right) J$ for some $z \in J$ and $s \geq 1$. Without loss of generality, we may replace $\mathbf{x}$ by $\mathbf{x}^{s}$ and assume $s=1$ :

$$
\zeta=\text { class of } z \bmod (\mathbf{x}) J \in H_{\mathfrak{m}}^{d}(J)=\lim _{\longrightarrow} \frac{J}{\left(\mathbf{x}^{t}\right) J} .
$$

Now, the natural map ${ }^{e} R \otimes_{R}\left(J /\left(\mathbf{x}^{t}\right) J\right) \rightarrow J \cdot{ }^{e} R /\left(\mathbf{x}^{t}\right) J \cdot{ }^{e} R=J^{[q]} /\left(\mathbf{x}^{q t}\right) J^{[q]}$ induces a map

$$
{ }^{e} R \otimes_{R} H_{\mathfrak{m}}^{d}(J) \rightarrow \underline{\lim } \frac{J^{[q]}}{\left(\mathbf{x}^{q t}\right) J^{[q]}},
$$

which sends $\zeta^{q}$ to the class of $z^{q} \bmod \left(\mathbf{x}^{q}\right) J^{[q]} \in J^{[q]} /\left(\mathbf{x}^{q}\right) J^{[q]}$. So, for $q=p^{e} \gg 0$, the class of $d z^{q} \bmod \left(\mathbf{x}^{q}\right) J^{[q]}$ in $\lim J^{[q]} /\left(\mathbf{x}^{q t}\right) J^{[q]}$ is zero, whence

$$
d z^{q} \in \mathcal{K}\left(\mathbf{x}^{q}, \infty, J^{[q]}\right) .
$$

By our assumption, this implies that

$$
c d z^{q} \in \mathcal{K}\left(\mathbf{x}^{q}, t_{0}, J^{[q]}\right)=\operatorname{Ker}\left(\frac{J^{[q]}}{\left(\mathbf{x}^{q}\right) J^{[q]}} \stackrel{\left(x_{1} \cdots x_{d}\right)^{q\left(t_{0}-1\right)}}{\longrightarrow} \frac{J^{[q]}}{\left(\mathbf{x}^{q t_{0}}\right) J^{[q]}}\right) .
$$

Hence one has

$$
c d z^{q}\left(x_{1} \cdots x_{d}\right)^{q\left(t_{0}-1\right)} \in\left(\mathbf{x}^{q t_{0}}\right) J^{[q]}=\left(\left(\mathbf{x}^{t_{0}}\right) J\right)^{[q]}
$$

for all $q=p^{e} \gg 0$. Thus $z\left(x_{1} \cdots x_{d}\right)^{t_{0}-1} \in\left(\left(\mathbf{x}^{t_{0}}\right) J\right)^{*}$, from which it follows that

$$
\zeta=\text { class of } z\left(x_{1} \cdots x_{d}\right)^{t_{0}-1} \bmod \left(\mathbf{x}^{t_{0}}\right) J \in 0_{H_{\mathfrak{m}}^{d}(J)}^{* f g} .
$$


Lemma A.3 (cf. $[\mathrm{Mc}]$ ). Suppose we are given an exact sequence of finitely generated $R$-modules $0 \rightarrow A \rightarrow B \rightarrow C \rightarrow 0$ satisfying the following condition: There exist $c_{1}, c_{2} \in R^{\circ}$ and $t_{1}, t_{2} \geq 1$ such that $c_{1} \cdot \operatorname{Image}\left(H^{d-1}\left(\mathbf{x}^{t} ; C\right) \rightarrow H^{d-1}\left(\mathbf{x}^{t+t_{1}} ; C\right)\right)=$ 0 for all $t \geq 1$, and that $c_{2} \mathcal{K}(\mathbf{x}, \infty, B) \subseteq \mathcal{K}\left(\mathbf{x}, t_{2}, B\right)$. Then

$$
c_{1} c_{2} \mathcal{K}(\mathbf{x}, \infty, A) \subseteq \mathcal{K}\left(\mathbf{x}, t_{1}+t_{2}, A\right) .
$$

Lemma A.4 ([Wi, Lemma 4.3]). Let $R$ be a normal domain, $J \subseteq R$ a divisorial ideal, and let $x_{1} \in J$. Then there exist an element $x_{2} \in R$ which is not in any minimal prime divisor of $x_{1}$ and $c \in J$ such that $x_{2}^{n} J^{(n)} \subseteq c^{n} R$ for all $n>0$.

Proof of Theorem [1.9] Let $r>0$ be the order of $\operatorname{cl}(J) \in \mathrm{Cl}(R)$, and let $J^{(r)}=x_{1} R$. By Lemma A.4, there exist $x_{2} \in R$ and $0 \neq c \in J$ such that $x_{2}^{n} J^{(n)} \subseteq c^{n} R$ for all $n>0$, and $x_{1}, x_{2}$ extends to a system of parameters $\mathbf{x}=x_{1}, x_{2}, \ldots, x_{d}$ of $R$.

Now, given any power $q=p^{e}$, write $q=k r+i$ for integers $k$ and $i$ with $0 \leq i \leq$ $r-1$. Then we have

$$
c^{r} x_{2}^{q} J^{(k r)} \subseteq c^{r} x_{2}^{k r} J^{(k r)} \subseteq c^{r+k r} R \subseteq c^{q} R \subseteq J^{[q]} .
$$

Since $x_{1}^{q} \in J^{[q]}$, this implies $c^{r}\left(x_{1} \cdots x_{i-1} x_{i+1} \cdots x_{d}\right)^{q s} J^{(k r)} \subseteq J^{[q]}$ for $s \geq 1$ and $1 \leq i \leq d$. Therefore, letting $c_{1}=c^{r}$, we have

$$
c_{1} \cdot \operatorname{Image}\left(H^{d-1}\left(\mathbf{x}^{q s t} ; J^{(k r)} / J^{[q]}\right) \rightarrow H^{d-1}\left(\mathbf{x}^{q s t+q s} ; J^{(k r)} / J^{[q]}\right)\right)=0
$$

for all $s, t \geq 1$. On the other hand, let $c_{2} \in R^{\circ}$ be a test element of $R$, which does exist by Theorem 1.12 If $z \in R$ is an element such that $z \bmod \left(\mathbf{x}^{q s}\right) \in \mathcal{K}\left(\mathbf{x}^{q s}, \infty, R\right)$, then $z \in\left(x_{1}^{q s t}, \ldots, x_{d}^{q s t}\right):\left(x_{1} \cdots x_{d}\right)^{q s(t-1)}$ for some $t \geq 1$, so $z \in\left(x_{1}^{q s}, \ldots, x_{d}^{q s}\right)^{*}$ by colon capturing [HH1]. This implies that

$$
c_{2} \mathcal{K}\left(\mathbf{x}^{q s}, \infty, J^{(k r)}\right) \cong c_{2} \mathcal{K}\left(\mathbf{x}^{q s}, \infty, R\right)=0 .
$$

Thus, applying Lemma A.3 to the exact sequence

$$
0 \rightarrow J^{[q]} \rightarrow J^{(k r)} \rightarrow J^{(k r)} / J^{[q]} \rightarrow 0,
$$

we see that

$$
c_{1} c_{2} \mathcal{K}\left(\mathbf{x}^{q s}, \infty, J^{[q]}\right) \subseteq \mathcal{K}\left(\mathbf{x}^{q s}, 2, J^{[q]}\right)
$$

for all $s \geq 1$ and $q=p^{e}$. Thanks to Theorem A.2, the proof is complete.

\section{REFERENCES}

[AM] I. Aberbach and B. MacCrimmon, Some results on test ideals, Proc. Edinburgh Math. Soc. (2) 42 (1999), 541-549. CMP 2000:04

[AN] Y. Akizuki and S. Nakano, Note on Kodaira-Spencer's proof of Lefschetz's theorem, Proc. Japan Acad. 30 (1954), 266-272. MR 16:619a

[D] M. Demazure, Anneaux gradués normaux, in "Introduction à la Théorie des Singularités II," Travaux en Cours 37, pp. 35-68, Hermann, Paris (1988). MR 91k:14004

[E] L. Ein, Multiplier ideals, vanishing theorems and applications, in "Algebraic GeometrySanta Cruz 1995," Proc. Symp. Pure Math. 62 (1997), Part I, 203-219. [MR 98m:14006

[EV] H. Esnault and E. Viehweg, "Lectures on Vanishing Theorems," DMV Seminar, Band 20, Birkhäuser Verlag, Basel (1992). MR 94a:14007

[F] R. Fedder, A Frobenius characterization of rational singularity in 2-dimensional graded rings, Trans. Amer. Math. Soc. 340 (1993), 655-668. MR 94b:13003

[FW] R. Fedder and K.-i. Watanabe, A characterization of F-regularity in terms of F-purity, in "Commutative Algebra," Math. Sci. Res. Inst. Publ. 15, pp. 227-245, Springer Verlag, New York (1989). MR 91k:13009

[GR] H. Grauert and O. Riemenschneider, Verschwindungssätze für analytische Kohomologiegruppen auf komplexen Räumen, Invent. Math. 11 (1970), 263-292. MR 46:2081 
[Ha] N. Hara, A characterization of rational singularities in terms of injectivity of Frobenius maps, Amer. J. Math. 120 (1998), 981-996. MR 99h:13005

[HW] N. Hara and K.-i. Watanabe, The injectivity of Frobenius acting on cohomology and local cohomology modules, Manuscripta Math. 90 (1996), 301-315. MR 97i:13016

[Ht] R. Hartshorne, "Residues and Duality," Lect. Notes in Math. 20, Springer Verlag, Heidelberg (1966). MR 36:5145

[HH1] M. Hochster and C. Huneke, Tight closure, invariant theory and the Briançon-Skoda theorem, J. Amer. Math. Soc. 3 (1990), 31-116. MR 91g:13010

[HH2] , Tight closure and strong F-regularity, Mem. Soc. Math. France 38 (1989), 119133. MR 91i:13025

[HH3] , Tight closures of parameter ideals and splitting in module-finite extensions, J. Alg. Geom. 3 (1994), 599-670. MR 95k:13002

[HR] M. Hochster and J. L. Roberts, The purity of the Frobenius and local cohomology, Adv. Math. 21 (1976), 117-172. MR 54:5230

[Hu] C. Huneke, "Tight closure and its applications," CBMS Regional Conf. Ser. Math. 88, Amer. Math. Soc., Providence (1996). MR 96m:13001

[HS] C. Huneke and K. E. Smith, Tight closure and the Kodaira vanishing theorem, J. Reine Angew. Math. 484 (1997), 127-152. MR 98e:13007

$[\mathrm{KMM}]$ Y. Kawamata, K. Matsuda and K. Matsuki, Introduction to the minimal model problem, in "Algebraic Geometry, Sendai 1985," Advanced Studies in Pure Math. 10, pp. 283-360, Kinokuniya, Tokyo, and North-Holland, Amsterdam (1987). MR 89e:14015

[LT] J. Lipman and B. Teissier, Pseudo-rational local rings and a theorem of Briançon-Skoda on the integral closure of ideals, Michigan Math. J. 28 (1981), 97-116. MR 82f:14004

[LS1] G. Lyubeznik and K. E. Smith, Strong and weak F-regularity are equivalent for graded rings, Amer. J. Math. 121 (1999), 1279-1290. CMP 2000:05

[LS2] On the commutation of the test ideal with localization and completion, Trans. Amer. Math. Soc. (to appear)

$[\mathrm{Mc}] \quad$ B. MacCrimmon, Weak F-regularity is strong F-regularity for rings with isolated non- $\mathbb{Q}$ Gorenstein points, Trans. Amer. Math. Soc. (to appear).

[MS] V. B. Mehta and V. Srinivas, A characterization of rational singularities, Asian J. Math. 1 (1997), 249-278 MR 99e:13009

[N] A. Nadel, Multiplier ideal sheaves and the existence of Kähler-Einstein metrics of positive scalar curvature, Proc. Nat. Acad. Sci. USA 86 (1990), 7299-7300. MR 90k:32061

[NW] K. Nakakoshi and K.-i. Watanabe, in preparation.

[S1] K. E. Smith, Test ideals in local rings, Trans. Amer. Math. Soc. 347 (1995), 3453-3472. MR 96c:13008

[S2] , F-rational rings have rational singularities, Amer. J. Math. 119 (1997), 159-180. MR 97k:13004

[S3] - The multiplier ideal is a universal test ideal, preprint.

[TW] M. Tomari and K.-i. Watanabe, Normal $\mathbb{Z}_{r}$-graded rings and normal cyclic covers, Manuscripta Math. 76 (1992), 325-340. MR 93j:13002

[W1] K.-i. Watanabe, Rational singularities with $k^{*}$-action, in "Commutative algebra, Proc. Trento Conference," Lect. Notes in Pure and Appl. Math. 84, pp. 331-359, Marcel Dekker (1983). MR 84e:14005

[W2] _ F-regular and F-pure normal graded rings, J. Pure Appl. Alg. 71 (1991), 341-350. MR 92g:13003

[W3] , F-regular and F-pure rings vs. log-terminal and log-canonical singularities, preprint.

[Wi] L. J. Williams, Uniform stability of kernels of Koszul cohomology indexed by the Frobenius endomorphism, J. Algebra 172 (1995), 721-743. MR 96f:13003

Department of Mathematical Sciences, Waseda University, Okubo, Shinjuku, Tokyo 169-8555, JAPAN

Current address: Mathematical Institute, Tohoku University, Sendai 980-8578, Japan

E-mail address: hara@math.tohoku.ac.jp 